\title{
ON THE INVERSE SCATTERING PROBLEM FOR JACOBI MATRICES WITH THE SPECTRUM ON AN INTERVAL, A FINITE SYSTEM OF INTERVALS OR A CANTOR SET OF POSITIVE LENGTH
}

\author{
A. Volberg And P. Yuditskit
}

\begin{abstract}
Solving inverse scattering problem for a discrete Sturm-Liouville operator with a rapidly decreasing potential one gets reflection coefficients $s_{ \pm}$and invertible operators $I+\mathcal{H}_{s_{ \pm}}$, where $\mathcal{H}_{s_{ \pm}}$is the Hankel operator related to the symbol $s_{ \pm}$. The MarchenkoFaddeev theorem (in the continuous case) [6] and the Guseinov theorem (in the discrete case) [4], guarantees the uniqueness of solution of the inverse scattering problem. In this article we ask the following natural question - can one find a precise condition guaranteeing that the inverse scattering problem is uniquely solvable and that operators $I+\mathcal{H}_{s_{ \pm}}$are invertible? Can one claim that uniqueness implies invertibility or vise versa?

Moreover we are interested here not only in the case of decreasing potential but also in the case of asymptotically almost periodic potentials. So we merge here two mostly developed cases of inverse problem for Sturm-Liouville operators: the inverse problem with (almost) periodic potential and the inverse problem with the fast decreasing potential.
\end{abstract}

\section{MAIN RESULTS}

Asymptotics of polynomials orthogonal on a homogeneous set, we described earlier [8], indicated strongly that there should be a scattering theory for Jacobi matrix with almost periodic background like it exists in the classical case of a constant background. Note that here left and right asymptotics are not necessary the same almost periodic coefficient sequences, but they are of the same spectral class. In this work we present all important ingredients of such theory: reflection/transmission coefficients, GelfandLevitan-Marchenko transformation operators, a Riemann-Hilbert problem related to the inverse scattering problem. At last now we can say that the reflectionless Jacobi matrices with homogeneous spectrum are those whose reflection coefficient is zero.

Moreover, we extend theory in depth and show that a reflection coefficient determine uniquely a Jacobi matrix of the Szegö class and both transformation operators are invertible if and only if the spectral density satisfies matrix $A_{2}$ condition [10].

Concerning $A_{2}$ condition in the inverse scattering we have to mention, at least as indirect references, the book [7, Chapter 2, Sect. 4] and the paper [1]. Generally references to stationary scattering and inverse scattering problems in connections with spatial 
asymptotics can be found in [3], where explicit expressions of transmission and reflection coefficients in terms of Weyl functions and phases asymptotic wave functions was given.

Let $J$ be a Jacobi matrix defining a bounded self-adjoint operator on $l^{2}(\mathbb{Z})$ :

$$
J e_{n}=p_{n} e_{n-1}+q_{n} e_{n}+p_{n+1} e_{n+1}, \quad n \in \mathbb{Z},
$$

where $\left\{e_{n}\right\}$ is the standard basis in $l^{2}(\mathbb{Z}), p_{n}>0$. The resolvent matrix-function is defined by the relation

$$
R(z)=R(z, J)=\mathcal{E}^{*}(J-z)^{-1} \mathcal{E}
$$

where $\mathcal{E}: \mathbb{C}^{2} \rightarrow l^{2}(\mathbb{Z})$ in such a way that

$$
\mathcal{E}\left[\begin{array}{c}
c_{-1} \\
c_{0}
\end{array}\right]=e_{-1} c_{-1}+e_{0} c_{0}
$$

This matrix-function possesses an integral representation

$$
R(z)=\int \frac{d \sigma}{x-z}
$$

with a $2 \times 2$ matrix-measure having a compact support on $\mathbb{R}$. $J$ is unitary equivalent to the operator multiplication by independent variable on

$$
L_{d \sigma}^{2}=\left\{f=\left[\begin{array}{c}
f_{-1}(x) \\
f_{0}(x)
\end{array}\right]: \int f^{*} d \sigma f<\infty\right\}
$$

The spectrum of $J$ is called absolutely continuous if the measure $d \sigma$ is absolutely continuous with respect to the Lebesgue measure on the real axis,

$$
d \sigma(x)=\rho(x) d x .
$$

Let $J_{0}$ be a Jacobi matrix with constant coefficients, $p_{n}=1, q_{n}=0$ (so called Chebyshev matrix). It has the following functional representation, besides the general one mentioned above. Note that the resolvent set of $J_{0}$ is the domain $\overline{\mathbb{C}} \backslash[-2,2]$. Let $z(\zeta): \mathbb{D} \rightarrow \overline{\mathbb{C}} \backslash[-2,2]$ be a uniformization of this domain, $z(\zeta)=1 / \zeta+\zeta$. With respect to the standard basis $\left\{t^{n}\right\}_{n \in \mathbb{Z}}$ in

$$
L^{2}=\left\{f(t): \int_{\mathbb{T}}|f|^{2} d m\right\}
$$

the matrix of the operator of multiplication by $z(t), t \in \mathbb{T}$, is the Jacobi matrix $J_{0}$, since $z(t) t^{n}=t^{n-1}+t^{n+1}$.

The famous Bernstein-Szeqö theorem implies the following proposition. 
Proposition 0.1. Let $J$ be a Jacobi matrix whose spectrum is an interval $[-2,2]$. Assume that the spectrum is absolutely continuous and the density of the spectral measure satisfies the condition

$$
\log \operatorname{det} \rho(z(t)) \in L^{1} .
$$

Then

$$
p_{n} \rightarrow 1, q_{n} \rightarrow 0, \quad n \rightarrow \pm \infty .
$$

Moreover, there exist generalized eigenvectors

$$
\begin{aligned}
& p_{n} e^{+}(n-1, t)+q_{n} e^{+}(n, t)+p_{n+1} e^{+}(n+1, t)=z(t) e^{+}(n, t) \\
& p_{n} e^{-}(-n, t)+q_{n} e^{-}(-n-1, t)+p_{n+1} e^{-}(-n-2, t)=z(t) e^{-}(-n-1, t)
\end{aligned}
$$

such that the following asymptotics hold true

$$
\begin{aligned}
& s(t) e^{ \pm}(n, t)=s(t) t^{n}+o(1), \quad n \rightarrow+\infty \\
& s(t) e^{ \pm}(n, t)=t^{n}+s_{\mp}(t) t^{-n-1}+o(1), \quad n \rightarrow-\infty
\end{aligned}
$$

in $L^{2}$.

To clarify the meaning of the words "generalized eigenvectors" we need some definitions and notation.

The matrix

$$
S(t)=\left[\begin{array}{cc}
s_{-} & s \\
s & s_{+}
\end{array}\right](t)
$$

is called the scattering matrix-function. It is a unitary-valued matrix-function with the following symmetry property:

$$
S^{*}(\bar{t})=S(t),
$$

and the analytic property:

$$
s(t) \text { is boundary values of an outer function. }
$$

We still denote by $s(\zeta), \zeta \in \mathbb{D}$, the values of the function inside the disk, and in the sequel, we assume that $s$ meets the normalization condition $s(0)>0$.

In fact, these mean that each of the entries $s_{ \pm}$(so called reflection coefficient) determines the matrix $S(t)$ in unique way. Indeed, since

$$
|s(t)|^{2}+\left|s_{ \pm}(t)\right|^{2}=1,
$$

using (0.11), we have

$$
s(\zeta)=e^{\frac{1}{2} \int_{\mathbb{T}} \frac{t+\zeta}{t-\zeta} \log \left\{1-\left|s_{ \pm}(t)\right|^{2}\right\} d m} .
$$

Then, we can solve for $s_{\mp}$ the relation

$$
\bar{s}_{+} s+\bar{s} s_{-}=0 .
$$


With the function $s_{ \pm}$we associate the metric

$$
\begin{aligned}
\|f\|_{s_{ \pm}}^{2} & =\frac{1}{2}\left\langle\left[\begin{array}{cc}
1 & \overline{s_{ \pm}(t)} \\
s_{ \pm}(t) & 1
\end{array}\right]\left[\begin{array}{c}
f(t) \\
\bar{t} f(\bar{t})
\end{array}\right],\left[\begin{array}{c}
f(t) \\
\bar{t} f(\bar{t})
\end{array}\right]\right\rangle \\
& =\left\langle f(t)+\bar{t}\left(s_{ \pm} f\right)(\bar{t}), f(t)\right\rangle, \quad f \in L^{2}
\end{aligned}
$$

Note that the conditions $(0.11),(0.12)$ guarantee that $\|f\|_{s_{ \pm}}=0$ implies $f=0$. We denote by $L_{d m, s_{ \pm}}^{2}$ or $L_{s_{ \pm}}^{2}$ (for shortness) the closer of $L^{2}$ with respect to this new metric.

The following relation sets a unitary map from $L_{s_{+}}^{2}$ to $L_{s_{-}}^{2}$ :

$$
s(t) f^{-}(t)=\bar{t} f^{+}(\bar{t})+s_{+}(t) f^{+}(t)
$$

moreover, in this case,

$$
\left\|f^{+}\right\|_{s_{+}}^{2}=\left\|f^{-}\right\|_{s_{-}}^{2}=\frac{1}{2}\left\{\left\|s f^{+}\right\|^{2}+\left\|s f^{-}\right\|^{2}\right\}
$$

and the inverse map is of the form

$$
s(t) f^{+}(t)=\bar{t} f^{-}(\bar{t})+s_{-}(t) f^{-}(t) .
$$

We say that a Jacobi matrix $J$ with the spectrum $[-2,2]$ is of Szegö class if its spectral measure $d \sigma$ satisfies $(0.4),(0.5)$.

Theorem 0.1. Let $J$ be a Jacobi matrix of Szegö class with the spectrum $E=[-2,2]$. Then there exists a unique unitary-valued matrix-function $S(t)$ of the form (0.9) possessing the properties (0.10), (0.11), and a unique pair of Fourier transforms

$$
\mathcal{F}^{ \pm}: l^{2}(\mathbb{Z}) \rightarrow L_{s_{ \pm}}^{2}, \quad\left(\mathcal{F}^{ \pm} J f\right)(t)=z(t)\left(\mathcal{F}^{ \pm} f\right)(t),
$$

determining each other by the relations

$$
s(t)\left(\mathcal{F}^{ \pm} f\right)(t)=\bar{t}\left(\mathcal{F}^{\mp} f\right)(\bar{t})+s_{\mp}(t)\left(\mathcal{F}^{\mp} f\right)(t),
$$

and possessing the following analytic properties

$$
s \mathcal{F}^{ \pm}\left(l^{2}\left(\mathbb{Z}_{ \pm}\right)\right) \subset H^{2}
$$

and the asymptotic properties

$$
e^{ \pm}(n, t)=t^{n}+o(1) \quad \text { in } L_{s_{ \pm}}^{2}, \quad n \rightarrow+\infty
$$

where

$$
e^{+}(n, t)=\left(\mathcal{F}^{+} e_{n}\right)(t), \quad e^{-}(n, t)=\left(\mathcal{F}^{-} e_{-n-1}\right)(t) .
$$

(As before, $\left\{e_{n}\right\}$ is the standard basis in $l^{2}(\mathbb{Z})$ ).

Remark 0.1. Show that (0.17) is equivalent to (0.8). Due to

$$
\left[\begin{array}{cc}
1 & \bar{s}_{ \pm} \\
s_{ \pm} & 1
\end{array}\right]=\left[\begin{array}{cc}
|s|^{2} & 0 \\
0 & 0
\end{array}\right]+\left[\begin{array}{c}
\bar{s}_{ \pm} \\
1
\end{array}\right]\left[\begin{array}{ll}
s_{ \pm} & 1
\end{array}\right]
$$


(0.17) is equivalent to $(n \rightarrow+\infty)$

$$
\begin{aligned}
& s(t) e^{ \pm}(n, t)=s(t) t^{n}+o(1) \quad \text { in } L^{2}, \\
& s_{ \pm}(t) e^{ \pm}(n, t)+\bar{t} e^{ \pm}(n, \bar{t})=s_{ \pm}(t) t^{n}+\bar{t}^{n+1}+o(1) \quad \text { in } L^{2} .
\end{aligned}
$$

Using (0.15), we rewrite the second relation into the form

$$
s(t) e^{\mp}(-n-1, t)=t^{-n-1}+s_{ \pm}(t) t^{n}+o(1) \quad \text { in } L^{2} .
$$

Substituting $n:=-n-1$, we get the second relation of $(0.8)$.

A fundamental question is how to recover the Jacobi matrix from the scattering matrix, in fact, from the reflection coefficient $s_{+}$(or $\left.s_{-}\right)$? When can this be done? Do we have a uniqueness theorem?

We show that for an arbitrary function $s_{+}(t)$ satisfying

$$
\overline{s_{+}(\bar{t})}=s_{+}(t) \quad \text { and } \quad \log \left\{1-\left|s_{+}(t)\right|^{2}\right\} \in L^{1},
$$

there exists a Jacobi matrix $J$ of Szegö class such that $s_{+}(t)$ is its reflection coefficient. But we can construct a matrix with this property, at least, in two different ways.

First, consider the space

$$
H_{s_{+}}^{2}=\operatorname{clos}_{L_{s_{+}}^{2}} H^{2},
$$

and introduce the Hankel operator $\mathcal{H}_{s_{+}}: H^{2} \rightarrow H^{2}$,

$$
\mathcal{H}_{s_{+}} f=P_{+} \bar{t}\left(s_{+} f\right)(\bar{t}), \quad f \in H^{2},
$$

where $P_{+}$is the Riesz projection from $L^{2}$ onto $H^{2}$. This operator determines the metric in $H_{s_{+}}^{2}$ :

$$
\begin{aligned}
\|f\|_{s_{+}}^{2} & =\left\langle f(t)+\bar{t}\left(s_{+} f\right)(\bar{t}), f(t)\right\rangle \\
& =\left\langle\left(I+\mathcal{H}_{s_{+}}\right) f, f\right\rangle, \quad \forall f \in H^{2} .
\end{aligned}
$$

Lemma 0.1. Under the assumptions (0.19), the space $H_{s_{+}}^{2}$ is a space of holomorphic functions with a reproducing kernel. Moreover, $s f \in H^{2}$ for any $f \in H_{s_{+}}^{2}$, and the reproducing vector $k_{s_{+}}$:

$$
\left\langle f, k_{s_{+}}\right\rangle=f(0), \quad \forall f \in H_{s_{+}}^{2},
$$

is of the form

$$
k_{s_{+}}=\left(I+\mathcal{H}_{s_{+}}\right)^{[-1]} \mathbf{1}:=\lim _{\epsilon \rightarrow 0^{+}}\left(\epsilon+I+\mathcal{H}_{s_{+}}\right)^{-1} \mathbf{1} \quad \text { in } L_{s_{+}}^{2} .
$$

Put $K_{s_{+}}(t)=k_{s_{+}}(t) / \sqrt{k_{s_{+}}(0)}$. 
Theorem 0.2. Let $s_{+}(t)$ satisfy (0.19). Then the system of functions $\left\{t^{n} K_{s_{+} t^{2 n}}(t)\right\}_{n \in \mathbb{Z}}$ forms an orthonormal basis in $L_{s_{+}}^{2}$. With respect to this basis, operator multiplication by $z(t)$ is a Jacobi matrix $J$ of Szegö class. Moreover, the initial function $s_{+}(t)$ is the reflection coefficient of the scattering matrix-function $S(t)$, associated to $J$ by Theorem 0.1 , and

$$
e^{+}(n, t)=t^{n} K_{s_{+} t^{2 n}}(t)
$$

From the other hand, the system of functions $\left\{t^{n} K_{s_{-}} t^{2 n}(t)\right\}_{n \in \mathbb{Z}}$ forms an orthonormal basis in $L_{s_{-}}^{2}$, and we are able to define a Jacobi matrix $\tilde{J}$ by the relation

$$
z(t) \tilde{e}^{+}(n, t)=\tilde{p}_{n} \tilde{e}^{+}(n-1, t)+\tilde{q}_{n} \tilde{e}^{+}(n, t)+\tilde{p}_{n+1} \tilde{e}^{+}(n+1, t)
$$

where $\left\{\tilde{e}^{+}(n, t)\right\}$ is the dual system to the system $\left\{t^{n} K_{s_{-}} t^{2 n}(t)\right\}$ (see $(0.15)$ ), i.e.:

$$
s(t) \tilde{e}^{+}(-n-1, t)=\bar{t}^{n+1} K_{s_{-} t^{2 n}}(\bar{t})+s_{-}(t) t^{n} K_{s_{-} t^{2 n}}(t) .
$$

Even the invertibility condition for the operators $\left(I+\mathcal{H}_{s_{ \pm}}\right)$does not guarantee that operators $J$ and $\tilde{J}$ are the same. But if $J=\tilde{J}$, then the uniqueness theorem takes place.

Theorem 0.3. Let $s_{+}$satisfy (0.19). Then the reflection coefficient $s_{+}$determines $a$ Jacobi matrix $J$ of Szegö class in a unique way if and only if the following relations take place

$$
s(0) K_{s_{ \pm}}(0) K_{s_{\mp} t^{-2}}(0)=1 .
$$

Corollary 0.1. Let $J$ be a Jacobi matrix of Szegö class with the spectrum $[-2,2]$ and let $\rho$ be the density of its spectral measure. If

$$
\int_{-2}^{2} \rho^{-1}(x) d x<\infty
$$

then there is no other Jacobi matrix of Szegö class with the same scattering matrix-function $S(t)$.

It is important to know, when the operators $\left(I+\mathcal{H}_{s_{ \pm}}\right)$, playing a central role in the inverse scattering problem, are invertible in the proper sense of words.

Theorem 0.4. Let $J$ be a Jacobi matrix of Szeqö class with the spectrum $[-2,2]$. Let $\rho$ be the density of its spectral measure and let $s_{+}$be the reflection coefficient of its scattering matrix-function. Then the following statements are equivalent.

1. The spectral density $\rho$ satisfies condition $A_{2}$.

2. The reflection coefficient $s_{+}$determines a Jacobi matrix of Szeqö class uniquely and both operators $\left(I+\mathcal{H}_{s_{ \pm}}\right)$are invertible.

To extend these results to the case when a spectrum $E$ is a finite system of intervals or a Cantor set of positive measure, we need only to introduce a counterpart of Hardy space. 
Let $z(\zeta): \mathbb{D} \rightarrow \Omega$ be a uniformization of the domain $\Omega=\overline{\mathbb{C}} \backslash E$. Thus there exists a discrete subgroup $\Gamma$ of the group $S U(1,1)$ consisting of elements of the form

$$
\gamma=\left[\begin{array}{ll}
\gamma_{11} & \gamma_{12} \\
\gamma_{21} & \gamma_{22}
\end{array}\right], \gamma_{11}=\overline{\gamma_{22}}, \gamma_{12}=\overline{\gamma_{21}}, \operatorname{det} \gamma=1,
$$

such that $z(\zeta)$ is automorphic with respect to $\Gamma$, i.e., $z(\gamma(\zeta))=z(\zeta), \forall \gamma \in \Gamma$, and any two preimages of $z_{0} \in \Omega$ are $\Gamma$-equivalent, i.e.,

$$
z\left(\zeta_{1}\right)=z\left(\zeta_{2}\right) \Rightarrow \exists \gamma \in \Gamma: \zeta_{1}=\gamma\left(\zeta_{2}\right) .
$$

We normalize $z(\zeta)$ by the conditions $z(0)=\infty,(\zeta z)(0)>0$.

A character of $\Gamma$ is a complex-valued function $\alpha: \Gamma \rightarrow \mathbb{T}$, satisfying

$$
\alpha\left(\gamma_{1} \gamma_{2}\right)=\alpha\left(\gamma_{1}\right) \alpha\left(\gamma_{2}\right), \quad \gamma_{1}, \gamma_{2} \in \Gamma .
$$

The characters form an Abelian compact group denoted by $\Gamma^{*}$.

For a given character $\alpha \in \Gamma^{*}$, as usual let us define

$$
H^{\infty}(\Gamma, \alpha)=\left\{f \in H^{\infty}: f(\gamma(\zeta))=\alpha(\gamma) f(\zeta), \forall \gamma \in \Gamma\right\} .
$$

Generally, a group $\Gamma$ is said to be of Widom type if for any $\alpha \in \Gamma^{*}$ the space $H^{\infty}(\Gamma, \alpha)$ is not trivial (contains a non-constant function).

A group of Widom type acts dissipatively on $\mathbb{T}$ with respect to $d m$, that is there exists a measurable (fundamental) set $\mathbb{E}$, which does not contain any two $\Gamma$-equivalent points, and the union $\cup_{\gamma \in \Gamma} \gamma(\mathbb{E})$ is a set of full measure. We can choose $\mathbb{E}$ possessing the symmetry property: $t \in \mathbb{E} \Rightarrow \bar{t} \in \mathbb{E}$. For the space of square summable functions on $\mathbb{E}$ (with respect to the Lebesgue measure), we use the notation $L_{d m \mid \mathbb{E}}^{2}$.

Let $f$ be an analytic function in $\mathbb{D}, \gamma \in \Gamma$ and $k \in \mathbb{N}$. Then we put

$$
f \mid[\gamma]_{k}=\frac{f(\gamma(\zeta))}{\left(\gamma_{21} \zeta+\gamma_{22}\right)^{k}} .
$$

Notice that $f \mid[\gamma]_{2}=f \forall \gamma \in \Gamma$, means that the form $f(\zeta) d \zeta$ is invariant with respect to the substitutions $\zeta \rightarrow \gamma(\zeta)(f(\zeta) d \zeta$ is an Abelian integral on $\mathbb{D} / \Gamma)$. Analogically, $f \mid[\gamma]=\alpha(\gamma) f \forall \gamma \in \Gamma$, means that the form $|f(\zeta)|^{2}|d \zeta|$ is invariant with respect to these substitutions.

We recall, that a function $f(\zeta)$ is of Smirnov class, if it can be represented as a ratio of two functions from $H^{\infty}$ with an outer denominator.

Definition. Let $\Gamma$ be a group of Widom type. The space $A_{1}^{2}(\Gamma, \alpha)\left(A_{2}^{1}(\Gamma, \alpha)\right)$ is formed by functions $f$, which are analytic on $\mathbb{D}$ and satisfy the following three conditions

1) $f$ is of Smirnov class

2) $f \mid[\gamma]=\alpha(\gamma) f \quad\left(f \mid[\gamma]_{2}=\alpha(\gamma) f\right) \quad \forall \gamma \in \Gamma$

3) $\int_{\mathbb{E}}|f|^{2} d m<\infty \quad\left(\int_{\mathbb{E}}|f| d m<\infty\right)$. 
$A_{1}^{2}(\Gamma, \alpha)$ is a Hilbert space with the reproducing kernel $k^{\alpha}\left(\zeta, \zeta_{0}\right)$, moreover

$$
0<\inf _{\alpha \in \Gamma^{*}} k^{\alpha}\left(\zeta_{0}, \zeta_{0}\right) \leq \sup _{\alpha \in \Gamma^{*}} k^{\alpha}\left(\zeta_{0}, \zeta_{0}\right)<\infty .
$$

Put

$$
k^{\alpha}(\zeta)=k^{\alpha}(\zeta, 0) \quad \text { and } \quad K^{\alpha}(\zeta)=\frac{k^{\alpha}(\zeta)}{\sqrt{k^{\alpha}(0)}} .
$$

We need one more special function. The Blaschke product

$$
b(\zeta)=\zeta \prod_{\gamma \in \Gamma, \gamma \neq 1_{2}} \frac{\gamma(0)-\zeta}{1-\overline{\gamma(0)} \zeta} \frac{|\gamma(0)|}{\gamma(0)}
$$

is called the Green's function of $\Gamma$ with respect to the origin. It is character-automorphic function, i.e., there exists $\mu \in \Gamma^{*}$ such that $b \in H^{\infty}(\Gamma, \mu)$. Note, if $G(z)=G(z, \infty)$ denotes the Green's function of the domain $\Omega$, then

$$
G(z(\zeta))=-\log |b(\zeta)| .
$$

Theorem [5]. Let $\Gamma$ be a group of Widom type. The following statements are equivalent: 1) The function $K^{\alpha}(0)$ is continuous on $\Gamma^{*}$.

2) $\sup \left\{|f(0)|: f \in H^{\infty}(\Gamma, \alpha),\|f\| \leq 1\right\} \rightarrow 1, \quad \alpha \rightarrow 1_{\Gamma^{*}}$.

3) The Direct Cauchy Theorem holds:

$$
\int_{\mathbb{E}} \frac{f}{b}(t) \frac{d t}{2 \pi i}=\frac{f}{b^{\prime}}(0), \quad \forall f \in A_{2}^{1}(\Gamma, \mu) .
$$

4) Let $\overline{t A_{1}^{2}\left(\Gamma, \alpha^{-1}\right)}=\left\{g=\overline{t f}: f \in A_{1}^{2}\left(\Gamma, \alpha^{-1}\right)\right\}$. Then

$$
L_{d m \mid \mathbb{E}}^{2}=\overline{t A_{1}^{2}\left(\Gamma, \alpha^{-1}\right)} \oplus A_{1}^{2}(\Gamma, \alpha) \quad \forall \alpha \in \Gamma^{*} .
$$

5) Every invariant subspace $M \subset A_{1}^{2}(\Gamma, \alpha)$ (i.e. $\phi M \subset M \forall \phi \in H^{\infty}(\Gamma)$ ) is of the form

$$
M=\Delta A_{1}^{2}\left(\Gamma, \beta^{-1} \alpha\right)
$$

for some character-automorphic inner function $\Delta \in H^{\infty}(\beta)$.

Definition [2]. A measurable set $E$ is homogeneous if there is an $\eta>0$ such that

$$
|(x-\delta, x+\delta) \cap E| \geq \eta \delta \quad \text { for all } 0<\delta<1 \quad \text { and all } x \in E .
$$

A Cantor set of positive length is an example of a homogeneous set. Let $E$ be a homogeneous set, then the domain $\Omega=\bar{C} \backslash E$ (respectively the group $\Gamma$ ) is of Widom type and the Direct Cauchy Theorem holds.

Recall, that a sequence of real numbers $\left\{p_{n}\right\} \in l^{\infty}(\mathbb{Z})$ is called uniformly almost periodic if the set of sequences $\left\{\left\{p_{n+l}\right\}, l \in \mathbb{Z}\right\}$ is a precompact in $l^{\infty}(\mathbb{Z})$. The general way to produce a sequence of this type looks as follows: let $\mathcal{G}$ be a compact Abelian group, and let $f(g)$ be a continuous function on $\mathcal{G}$, then

$$
p_{n}:=f\left(g_{0}+n g_{1}\right), \quad g_{0}, g_{1} \in \mathcal{G},
$$

is an almost periodic sequence. A Jacobi matrix is almost periodic if the coefficient sequences are almost periodic. We denote by $J(E)$ the class of almost periodic Jacobi matrices with absolutely continuous homogeneous spectrum $E$. In what follows the class $J(E)$ plays a role of Chebyshev matrix. In fact, if $E=[-2,2]$ then $J(E)=\left\{J_{0}\right\}$. 
Theorem [9]. Let $E$ be a homogeneous set. Let $z: \mathbb{D} \rightarrow \overline{\mathbb{C}} \backslash E$ be a uniformizing mapping. Then the systems of functions $\left\{b^{n} K^{\alpha \mu^{-n}}\right\}_{n \in \mathbb{Z}_{+}}$and $\left\{b^{n} K^{\alpha \mu^{-n}}\right\}_{n \in \mathbb{Z}}$ form an orthonormal basis in $A_{1}^{2}(\Gamma, \alpha)$ and in $L_{d m \mid \mathbb{E}}^{2}$, respectively, for any $\alpha \in \Gamma^{*}$. With respect to this basis, the operator multiplication by $z(t)$ is a three-diagonal almost periodic Jacobi matrix $J(\alpha)$. Moreover,

$$
J(E)=\left\{J(\alpha): \alpha \in \Gamma^{*}\right\},
$$

and $J(\alpha)$ is a continuous function on $\Gamma^{*}$.

We say that a Jacobi matrix $J$ with the spectrum $E$ is of Szegö class if its spectral measure is absolutely continuous, $d \sigma(x)=\rho(x) d x$, and $\rho(z(t))$ satisfies (0.5).

Theorem 0.5. Let $J$ be a Jacobi matrix of Szegö class with a homogeneous spectrum $E$. Then there exists a unique unitary-valued matrix-function $S(t)$ of the form (0.9) possessing the properties (0.10), (0.11), and a unique pair of Fourier transforms

$$
\mathcal{F}^{ \pm}: l^{2}(\mathbb{Z}) \rightarrow L_{d m \mid \mathbb{E}, s_{ \pm}}^{2}, \quad\left(\mathcal{F}^{ \pm} J f\right)(t)=z(t)\left(\mathcal{F}^{ \pm} f\right)(t),
$$

determining each other by the relations

$$
s(t)\left(\mathcal{F}^{ \pm} f\right)(t)=\bar{t}\left(\mathcal{F}^{\mp} f\right)(\bar{t})+s_{\mp}(t)\left(\mathcal{F}^{\mp} f\right)(t),
$$

and possessing the following analytic properties

$$
s \mathcal{F}^{ \pm}\left(l^{2}\left(\mathbb{Z}_{ \pm}\right)\right) \subset A_{1}^{2}\left(\Gamma, \alpha_{\mp}^{-1}\right),
$$

and the asymptotic properties

$$
e^{ \pm}(n, t)=b^{n}(t) K^{\alpha_{ \pm} \mu^{-n}}(t)+o(1) \quad \text { in } L_{d m \mid \mathbb{E}, s_{ \pm}}^{2}, \quad n \rightarrow+\infty,
$$

where

$$
e^{+}(n, t)=\left(\mathcal{F}^{+} e_{n}\right)(t), \quad e^{-}(n, t)=\left(\mathcal{F}^{-} e_{-n-1}\right)(t),
$$

and $L_{d m \mid \mathbb{E}, s_{ \pm}}^{2}$ is the closer of the functions from $L_{d m \mid \mathbb{E}}^{2}$ with respect to the metric

$$
\|f\|_{s_{ \pm}}^{2}=\frac{1}{2}\left\langle\left[\begin{array}{cc}
1 & \overline{s_{ \pm}(t)} \\
s_{ \pm}(t) & 1
\end{array}\right]\left[\begin{array}{c}
f(t) \\
\bar{t} f(\bar{t})
\end{array}\right],\left[\begin{array}{c}
f(t) \\
\bar{t} f(\bar{t})
\end{array}\right]\right\rangle, \quad f \in L_{d m \mid \mathbb{E}}^{2} .
$$

Theorems $0.2-0.4$ also have their closely parallel counterparts in the case when the spectrum is a homogeneous set.

\section{IN THE MODEL SPACE}

Let $E$ be a homogeneous set. Let $z(\zeta): \mathbb{D} / \Gamma \sim \overline{\mathbb{C}} \backslash E$ be a uniformization and $b(\zeta)$ be the Green's function. Throughout the paper we assume that $(b z)(0)=1$. Let $\mathbb{E} \subset \mathbb{T}$ be a symmetric fundamental set $(t \in \mathbb{E} \Rightarrow \bar{t} \in \mathbb{E})$.

With a function $s_{+}(t) \in L_{d m \mid \mathbb{E}}^{\infty}$ such that

$$
\overline{s_{+}(\bar{t})}=s_{+}(t) \text { and } 1-\left|s_{+}(t)\right|^{2}>0 \text { a.e. on } \mathbb{E},
$$

we associate the metric

$$
\begin{aligned}
\|f\|_{s_{+}}^{2} & =\frac{1}{2}\left\langle\left[\begin{array}{cc}
1 & \overline{s_{+}(t)} \\
s_{+}(t) & 1
\end{array}\right]\left[\begin{array}{c}
f(t) \\
\bar{t} f(\bar{t})
\end{array}\right],\left[\begin{array}{c}
f(t) \\
\bar{t} f(\bar{t})
\end{array}\right]\right\rangle \\
& =\left\langle f(t)+\bar{t}\left(s_{+} f\right)(\bar{t}), f(t)\right\rangle, \quad f \in L_{d m \mid \mathbb{E}}^{2} .
\end{aligned}
$$

Condition (1.1) guarantee that $\|f\|_{s_{+}}=0$ implies $f=0$. We denote by $L_{d m \mid \mathbb{E}, s_{+}}^{2}$ or $L_{s_{+}}^{2}$ (for shortness) the closer of $L_{d m \mid \mathbb{E}}^{2}$ with respect to this metric. 
Lemma 1.1. The operator multiplication by $z(t)$ in $L_{s_{+}}^{2}$ is unitary equivalent to the operator multiplication by $z(t)$ in $L_{d m \mid \mathbb{E}}^{2}$

Proof. Let us put

$$
\left[\begin{array}{c}
g(t) \\
\bar{t} g(\bar{t})
\end{array}\right]=\left[\begin{array}{cc}
1 & \overline{s_{+}(t)} \\
s_{+}(t) & 1
\end{array}\right]^{1 / 2}\left[\begin{array}{c}
f(t) \\
\bar{t} f(\bar{t})
\end{array}\right], \quad f \in L_{d m \mid \mathbb{E}}^{2} .
$$

In this case $\|f\|_{s_{+}}=\|g\|$. The system of identities

$$
\begin{aligned}
{\left[\begin{array}{cc}
1 & \overline{s_{+}(t)} \\
s_{+}(t) & 1
\end{array}\right]^{1 / 2}\left[\begin{array}{c}
(z f)(t) \\
\bar{t}(z f)(\bar{t})
\end{array}\right] } & =\left[\begin{array}{cc}
1 & \overline{s_{+}(t)} \\
s_{+}(t) & 1
\end{array}\right]^{1 / 2} z(t)\left[\begin{array}{c}
f(t) \\
\bar{t} f(\bar{t})
\end{array}\right] \\
& =z(t)\left[\begin{array}{c}
g(t) \\
\bar{t} g(\bar{t})
\end{array}\right]=\left[\begin{array}{c}
(z g)(t) \\
\bar{t}(z g)(\bar{t})
\end{array}\right]
\end{aligned}
$$

finishes the proof.

Let $\alpha_{+} \in \Gamma^{*}$. In what further, we assume that $s_{+} \in L^{\infty}\left(\Gamma, \alpha_{+}^{-2}\right)$ and

$$
\log \left(1-\left|s_{+}(t)\right|^{2}\right) \in L^{1} .
$$

We define an outer function $s, s(0)>0$, by the relation

$$
|s(t)|^{2}=1-\left|s_{+}(t)\right|^{2}, \quad t \in \mathbb{T} .
$$

It is a character-automorphic function such that $\overline{s(\bar{t})}=s(t)$. It is convenient to denote its character by $\alpha_{+}^{-1} \alpha_{-}^{-1}$, i.e., $s \in H^{\infty}\left(\Gamma, \alpha_{+}^{-1} \alpha_{-}^{-1}\right)$.

Let us discuss some properties of the space

$$
H_{s_{+}}^{2}\left(\alpha_{+}\right):=\operatorname{clos}_{L_{s_{+}}^{2}} A_{1}^{2}\left(\Gamma, \alpha_{+}\right) .
$$

First of all, we define "a Hankel operator" $\mathcal{H}_{s_{+}}: A_{1}^{2}\left(\Gamma, \alpha_{+}\right) \rightarrow A_{1}^{2}\left(\Gamma, \alpha_{+}\right)$,

$$
\mathcal{H}_{s_{+}} f=P_{A_{1}^{2}\left(\Gamma, \alpha_{+}\right)} \bar{t}\left(s_{+} f\right)(\bar{t}) \text {. }
$$

Note, that this operator, indeed, does not depend on "an analytical part" of its symbol, more precisely,

$$
\mathcal{H}_{\left(s_{+}+\epsilon\right)}=\mathcal{H}_{s_{+}}, \quad \forall \epsilon \in H^{\infty}\left(\Gamma, \alpha_{+}^{-2}\right) .
$$

Besides, in the classical case $E=[-2,2], \Gamma=\left\{1_{2}\right\}, \mathbb{E}=\mathbb{T}$, with a function

$$
s_{+}(t)=\sum_{n \in \mathbb{Z}} a_{n} t^{n}
$$

is associated the operator $\mathcal{H}_{s_{+}}: H^{2} \rightarrow H^{2}$ having the representation

$$
\mathcal{H}_{s_{+}}=\left[\begin{array}{llll}
a_{-1} & a_{-2} & a_{-3} & \cdots \\
a_{-2} & a_{-3} & \ldots & \\
a_{-3} & \cdots & & \\
\ldots & & &
\end{array}\right]
$$

with respect to the standard basis $\left\{t^{n}\right\}_{n \in \mathbb{Z}_{+}}$in $H^{2}$.

The operator $\mathcal{H}_{s_{+}}$determines the metric in $H_{s_{+}}^{2}\left(\alpha_{+}\right)$:

$$
\begin{aligned}
\|f\|_{s_{+}}^{2} & =\left\langle f(t)+\bar{t}\left(s_{+} f\right)(\bar{t}), f(t)\right\rangle \\
& =\left\langle\left(I+\mathcal{H}_{s_{+}}\right) f, f\right\rangle, \quad f \in A_{1}^{2}\left(\Gamma, \alpha_{+}\right) .
\end{aligned}
$$


Lemma 1.2. Under the assumptions (1.2), the space $H_{s_{+}}^{2}\left(\alpha_{+}\right)$is a space of holomorphic functions with a reproducing kernel. Moreover, $s f \in A_{1}^{2}\left(\Gamma, \alpha_{-}^{-1}\right)$ for any $f \in H_{s_{+}}^{2}\left(\alpha_{+}\right)$, and the reproducing vector $k_{s_{+}}^{\alpha_{+}}$:

$$
\left\langle f, k_{s_{+}}^{\alpha_{+}}\right\rangle=f(0), \quad \forall f \in H_{s_{+}}^{2}\left(\alpha_{+}\right),
$$

is of the form

$$
k_{s_{+}}^{\alpha_{+}}=\left(I+\mathcal{H}_{s_{+}}\right)^{[-1]} k^{\alpha_{+}}:=\lim _{\epsilon \rightarrow 0^{+}}\left(\epsilon+I+\mathcal{H}_{s_{+}}\right)^{-1} k^{\alpha_{+}} \quad \text { in } L_{s_{+}}^{2} .
$$

Proof. From the inequality

$$
\left[\begin{array}{cc}
1 & \overline{s_{+}(t)} \\
s_{+}(t) & 1
\end{array}\right]-\left[\begin{array}{cc}
|s(t)|^{2} & 0 \\
0 & 0
\end{array}\right]=\left[\begin{array}{cc}
\left|s_{+}(t)\right|^{2} & \overline{s_{+}(t)} \\
s_{+}(t) & 1
\end{array}\right] \geq 0,
$$

it follows that

$$
\|s f\|^{2} \leq 2\|f\|_{s_{+}}^{2} \quad \forall f \in L_{s_{+}}^{2} .
$$

Thus, if a sequence $\left\{f_{n}\right\}, f_{n} \in A_{1}^{2}\left(\Gamma, \alpha_{+}\right)$, converges in $H_{s_{+}}^{2}\left(\alpha_{+}\right)$, then the sequence $\left\{s f_{n}\right\}$ converges in $A_{1}^{2}\left(\Gamma, \alpha_{-}^{-1}\right)$. In the same way we have boundedness of the functional $f \rightarrow f(0)$,

$$
|f(0)|^{2} \leq \frac{1}{|s(0)|^{2}}|(s f)(0)|^{2} \leq\left.\frac{2}{|s(0)|^{2}}|| f\right|_{s_{+}} ^{2} k^{\alpha_{-}^{-1}}(0) .
$$

Let us prove (1.3). Let $\epsilon>0$, then for the norm of the difference we have an estimate

$$
\begin{aligned}
\| k_{s_{+}}^{\alpha_{+}} & -\left(\epsilon+I+\mathcal{H}_{s_{+}}\right)^{-1} k^{\alpha_{+}} \|_{s_{+}}^{2}=k_{s_{+}}^{\alpha_{+}}(0)-2\left\{\left(\epsilon+I+\mathcal{H}_{s_{+}}\right)^{-1} k^{\alpha_{+}}\right\}(0) \\
& +\left\langle\left(I+\mathcal{H}_{s_{+}}\right)\left(\epsilon+I+\mathcal{H}_{s_{+}}\right)^{-1} k^{\alpha_{+}},\left(\epsilon+I+\mathcal{H}_{s_{+}}\right)^{-1} k^{\alpha_{+}}\right\rangle \\
& \leq k_{s_{+}}^{\alpha_{+}}(0)-\left\{\left(\epsilon+I+\mathcal{H}_{s_{+}}\right)^{-1} k^{\alpha_{+}}\right\}(0) .
\end{aligned}
$$

Therefore,

$$
\left\{\left(\epsilon+I+\mathcal{H}_{s_{+}}\right)^{-1} k^{\alpha_{+}}\right\}(0) \leq k_{s_{+}}^{\alpha_{+}}(0) .
$$

Besides, (1.5) implies that (1.3) follows from the relation

$$
\lim _{\epsilon \rightarrow 0}\left\{\left(\epsilon+I+\mathcal{H}_{s_{+}}\right)^{-1} k^{\alpha_{+}}\right\}(0)=k_{s_{+}}^{\alpha_{+}}(0) .
$$

Let us prove (1.7). Since the function

$$
\left\{\left(\epsilon+I+\mathcal{H}_{s_{+}}\right)^{-1} k^{\alpha_{+}}\right\}(0)=\left\langle\left(\epsilon+I+\mathcal{H}_{s_{+}}\right)^{-1} k^{\alpha_{+}}, k^{\alpha_{+}}\right\rangle
$$

decreases with $\epsilon$ and it is bounded by (1.6), there exists a limit

$$
\lim _{\epsilon \rightarrow 0}\left\{\left(\epsilon+I+\mathcal{H}_{s_{+}}\right)^{-1} k^{\alpha_{+}}\right\}(0) \leq k_{s_{+}}^{\alpha_{+}}(0) .
$$


From the other hand, for any $f \in A_{1}^{2}\left(\Gamma, \alpha_{+}\right)$and $\epsilon>0$ the following inequalities hold

$$
\begin{aligned}
|f(0)|^{2} & \leq\left\langle\left(\epsilon+I+\mathcal{H}_{s_{+}}\right)^{-1} k^{\alpha_{+}}, k^{\alpha_{+}}\right\rangle\left\langle\left(\epsilon+I+\mathcal{H}_{s_{+}}\right) f, f\right\rangle \\
& \leq\left\{\lim _{\epsilon \rightarrow 0}\left\langle\left(\epsilon+I+\mathcal{H}_{s_{+}}\right)^{-1} k^{\alpha_{+}}, k^{\alpha_{+}}\right\rangle\right\}\left\langle\left(\epsilon+I+\mathcal{H}_{s_{+}}\right) f, f\right\rangle,
\end{aligned}
$$

that is

$$
|f(0)|^{2} \leq\left\{\lim _{\epsilon \rightarrow 0}\left\langle\left(\epsilon+I+\mathcal{H}_{s_{+}}\right)^{-1} k^{\alpha_{+}}, k^{\alpha_{+}}\right\rangle\right\}\|f\|_{s_{+}}^{2} .
$$

Putting $f=k_{s_{+}}^{\alpha_{+}}$, we have

$$
k_{s_{+}}^{\alpha_{+}}(0) \leq \lim _{\epsilon \rightarrow 0}\left\langle\left(\epsilon+I+\mathcal{H}_{s_{+}}\right)^{-1} k^{\alpha_{+}}, k^{\alpha_{+}}\right\rangle .
$$

Comparing this inequality with (1.8), we get (1.7), thus (1.3) is proved.

We define $s_{-} \in L^{\infty}\left(\Gamma, \alpha_{-}^{-2}\right)$ by

$$
s_{-}(t)=-\overline{s_{+}(t)} s(t) / \overline{s(t)} .
$$

In this case

$$
S(t)=\left[\begin{array}{cc}
s_{-} & s \\
s & s_{+}
\end{array}\right](t)
$$

is a unitary-valued matrix function possessing properties (0.10), (0.11).

Lemma 1.3. The following relation sets a unitary map from $L_{s_{+}}^{2}$ to $L_{s_{-}}^{2}$ :

$$
s(t) f^{-}(t)=\bar{t} f^{+}(\bar{t})+s_{+}(t) f^{+}(t) .
$$

Moreover, in this case,

$$
\left\|f^{+}\right\|_{s_{+}}^{2}=\left\|f^{-}\right\|_{s_{-}}^{2}=\frac{1}{2}\left\{\left\|s f^{+}\right\|^{2}+\left\|s f^{-}\right\|^{2}\right\},
$$

and the inverse map is of the form

$$
s(t) f^{+}(t)=\bar{t} f^{-}(\bar{t})+s_{-}(t) f^{-}(t) .
$$

Proof. These follow from the identities

$$
\left[\begin{array}{cc}
1 & \bar{s}_{+} \\
s_{+} & 1
\end{array}\right]=\left[\begin{array}{cc}
\bar{s}_{+} & 1 \\
1 & s_{+}
\end{array}\right]\left[\begin{array}{cc}
1 / \bar{s} & 0 \\
0 & 1 / s
\end{array}\right]\left[\begin{array}{cc}
1 & \bar{s}_{-} \\
s_{-} & 1
\end{array}\right]\left[\begin{array}{cc}
1 / s & 0 \\
0 & 1 / \bar{s}
\end{array}\right]\left[\begin{array}{cc}
s_{+} & 1 \\
1 & \bar{s}_{+}
\end{array}\right]
$$

and (0.18). 
Lemma 1.4. Let $K_{s_{+}}^{\alpha_{+}}(t)=k_{s_{+}}^{\alpha_{+}}(t) / \sqrt{k_{s_{+}}^{\alpha_{+}}(0)}$. The system of functions $\left\{b^{n}(t) K_{s_{+} b^{2 n}}^{\alpha_{+} \mu^{-n}}(t)\right\}$ forms an orthonormal basis in $H_{s_{+}}^{2}\left(\alpha_{+}\right)$when $\left\{n \in \mathbb{Z}_{+}\right\}$and in $L_{s_{+}}^{2}$ when $\{n \in \mathbb{Z}\}$. With respect to this basis the operator multiplication by $z(t)$ is a Jacobi matrix.

Proof. First, we note that

$$
\left\{f: f \in H_{s_{+}}^{2}\left(\alpha_{+}\right), f(0)=0\right\}=\left\{f=b \tilde{f}: \tilde{f} \in H_{s_{+} b^{2}}^{2}\left(\alpha_{+} \mu^{-1}\right)\right\} .
$$

Therefore,

$$
H_{s_{+}}^{2}\left(\alpha_{+}\right)=\left\{K_{s_{+}}^{\alpha_{+}}(t)\right\} \oplus b H_{s_{+} b^{2}}^{2}\left(\alpha_{+} \mu^{-1}\right) .
$$

Iterating this relation, we get that $\left\{b^{n}(t) K_{s_{+} b^{2 n}}^{\alpha_{+} \mu^{-n}}(t)\right\}_{n \in \mathbb{Z}_{+}}$is an orthonormal basis in $H_{s_{+}}^{2}\left(\alpha_{+}\right)$, since $\cap_{n \in \mathbb{Z}_{+}} b^{n} H_{s_{+} b^{2 n}}^{2}\left(\alpha_{+} \mu^{-n}\right)=\{0\}$.

Then, we note that an arbitrary function $f \in L_{s_{+}}^{2}$ can be approximated with the given accuracy by a function $f_{1}$ from $L_{d m \mid \mathbb{E}}^{2}$. This function, in its turn, can be approximated by a function $f_{2} \in b^{n} A_{1}^{2}\left(\Gamma, \alpha_{+} \mu^{-n}\right)$ with a suitable $n$. Therefore, linear combinations of functions from $\left\{b^{n}(t) K_{s_{+} b^{2 n}}^{\alpha_{+} \mu^{-n}}(t)\right\}$ are dense in $L_{s_{+}}^{2}$. Since this system of functions is orthonormal, it forms a basis in $L_{s_{+}}^{2}$.

Since $b z \in H^{\infty}(\Gamma, \mu)$, we have

$$
z: b^{n} H_{s_{+} b^{2 n}}^{2}\left(\alpha_{+} \mu^{-n}\right) \rightarrow b^{n-1} H_{s_{+} b^{2 n-2}}^{2}\left(\alpha_{+} \mu^{-n+1}\right) .
$$

For this reason, in the basis $\left\{b^{n}(t) K_{s_{+} b^{2 n}}^{\alpha_{+} \mu^{-n}}(t)\right\}_{n \in \mathbb{Z}}$, the matrix of the operator multiplication by $z(t)$ has only one non-zero entry over diagonal in each column. But the operator is self-adjoint, therefore, the matrix is a three-diagonal Jacobi matrix.

Lemma 1.5. Let $e^{+}(n, t)=b^{n}(t) K_{s_{+} b^{2 n}}^{\alpha_{+} \mu^{-n}}(t), n \in \mathbb{Z}$. Define

$$
s(t) e^{-}(n, t)=\bar{t} e^{+}(-n-1, \bar{t})+s_{+}(t) e^{+}(-n-1, t) .
$$

Then $\left\{e^{-}(n, t)\right\}$ is an orthonormal basis in $L_{s_{-}}^{2}$,

$$
s(t) e^{-}(n, t) \in A_{1}^{2}\left(\Gamma, \alpha_{+}^{-1}\right), \quad n \in \mathbb{Z}_{+},
$$

and

$$
e^{-}(0,0)\left(b e^{+}\right)(-1,0)=\frac{b^{\prime}(0)}{s(0)} .
$$

Proof. Lemma 1.3 and Lemma 1.4 imply immediately that $\left\{e^{-}(n, t)\right\}$ is an orthonormal basis in $L_{s_{-}}^{2}$. Moreover, $s(t) e^{-}(n, t) \in L_{d m \mid \mathbb{E}}^{2}$. To prove (1.9) consider a scalar product $\left(f \in A_{1}^{2}\left(\Gamma, \alpha_{+}\right)\right)$

$$
\begin{aligned}
\left\langle\bar{t} f(\bar{t}), s(t) e^{-}(n, t)\right\rangle & =\frac{1}{2}\left\langle\left[\begin{array}{c}
f(t) \\
\bar{t} f(\bar{t})
\end{array}\right],\left[\begin{array}{cc}
1 & \overline{s_{+}(t)} \\
s_{+}(t) & 1
\end{array}\right]\left[\begin{array}{l}
e^{-}(n, t) \\
\bar{t} e^{-}(n, \bar{t})
\end{array}\right]\right\rangle \\
& =\frac{1}{2}\left\langle\left[\begin{array}{c}
f(t) \\
\bar{t} f(\bar{t})
\end{array}\right],\left[\begin{array}{cc}
1 & \overline{s_{+}(t)} \\
s_{+}(t) & 1
\end{array}\right]\left[\begin{array}{c}
\left(b^{-n-1} K_{s_{+} b^{-2 n-2}}^{\alpha_{+}}\right)(t) \\
\bar{t}\left(b^{-n-1} K_{s_{+} b^{-2 n-2}}^{\alpha_{+}+1}\right)(\bar{t})
\end{array}\right]\right\rangle \\
& =\left\langle b^{n+1} f, K_{s_{+} b^{-2 n-2}}^{\alpha_{+} \mu^{n+1}}\right\rangle_{s_{+} b^{-2 n-2}}=0, \quad \forall n \geq 0 .
\end{aligned}
$$


To prove (1.10), we write

$$
s(0) e^{-}(0,0)=\left\langle s(t) e^{-}(0, t), k^{\alpha_{+}^{-1}}(t)\right\rangle .
$$

Due to the Direct Cauchy Theorem, the reproducing kernel $k^{\alpha}$ possesses the following property:

$$
\bar{t} k^{\alpha_{+}^{-1}}(\bar{t})=\frac{b^{\prime}(0)}{k^{\alpha_{+} \mu}(0)} \frac{k^{\alpha_{+} \mu}(t)}{b(t)} .
$$

Substituting (1.12) in (1.11), we obtain

$$
\begin{aligned}
s(0) e^{-}(0,0) & =\frac{b^{\prime}(0)}{2 k^{\alpha_{+} \mu}(0)}\left\langle\left[\begin{array}{cc}
1 & \overline{s_{+}(t)} \\
s_{+}(t) & 1
\end{array}\right]\left[\begin{array}{c}
e^{-}(-1, t) \\
\bar{t} e^{-}(-1, \bar{t})
\end{array}\right],\left[\begin{array}{c}
\left(b^{-1} k^{\alpha_{+} \mu}\right)(t) \\
\bar{t}\left(b^{-1} k^{\alpha_{+} \mu}\right)(\bar{t})
\end{array}\right]\right\rangle \\
& =\frac{b^{\prime}(0)}{k^{\alpha_{+} \mu}(0)}\left\langle K_{s_{+} b^{-2}}^{\alpha_{+}}(t), k^{\alpha_{+} \mu}(t)\right\rangle_{s_{+} b^{-2}} .
\end{aligned}
$$

Using (1.3), we have

$$
\begin{aligned}
s(0) e^{-}(0,0) & =\frac{b^{\prime}(0)}{k^{\alpha_{+} \mu}(0) K_{s_{+} b^{-2}}^{\alpha_{+}}(0)} \lim _{\epsilon \rightarrow 0}\left\langle\left(\epsilon+I+\mathcal{H}_{s_{+} b^{-2}}\right)^{-1} k^{\alpha_{+} \mu}, k^{\alpha_{+} \mu}\right\rangle_{s_{+} b^{-2}} \\
& =\frac{b^{\prime}(0)}{k^{\alpha_{+} \mu}(0) K_{s_{+} b^{-2}}^{\alpha_{+}}(0)} \lim _{\epsilon \rightarrow 0}\left\langle\left(I+\mathcal{H}_{s_{+} b^{-2}}\right)\left(\epsilon+I+\mathcal{H}_{s_{+} b^{-2}}\right)^{-1} k^{\alpha_{+} \mu}, k^{\alpha_{+} \mu}\right\rangle \\
& =\frac{b^{\prime}(0)}{k^{\alpha_{+} \mu}(0) K_{s_{+} b^{-2}}^{\alpha_{+}}(0)}\left\{k^{\alpha_{+} \mu}(0)-\lim _{\epsilon \rightarrow 0} \epsilon\left\langle\left(\epsilon+I+\mathcal{H}_{s_{+} b^{-2}}\right)^{-1} k^{\alpha_{+} \mu}, k^{\alpha_{+} \mu}\right\rangle\right\} .
\end{aligned}
$$

Since the limit (1.7) exists, finally, we get

$$
s(0) e^{-}(0,0)=\frac{b^{\prime}(0)}{K_{s_{+} b^{-2}}^{\alpha_{+}}(0)}=\frac{b^{\prime}(0)}{\left(b e^{+}\right)(-1,0)} .
$$

The lemma is proved.

Lemma 1.6. Let $\left\|s_{+}\right\|<1$. Then

$$
K_{s_{ \pm}}^{\alpha_{ \pm}}(0) K_{s_{\mp} b^{-2}}^{\alpha_{\mp} \mu}(0)=\frac{b^{\prime}(0)}{s(0)}
$$

Proof. Note, that operators $\left(I+\mathcal{H}_{s_{ \pm} b^{n}}\right)$ are invertible.

We use the notation of Lemma 1.5. As we know, $s(t) e^{-}(0, t) \in A_{1}^{2}\left(\Gamma, \alpha_{+}^{-1}\right)$. But, in the case under consideration, $1 / s \in H^{\infty}\left(\Gamma, \alpha_{+} \alpha_{-}\right)$. Hence, the function $e^{-}(0, t)$ itself belongs to $A_{1}^{2}\left(\Gamma, \alpha_{-}\right)$. Therefore, we can project each term onto $A_{1}^{2}\left(\Gamma, \alpha_{-}\right)$in the relation

$$
\bar{t}\left(s e^{+}\right)(-1, \bar{t})=e^{-}(0, t)+\bar{t}\left(s_{-} e^{-}\right)(0, \bar{t}) .
$$


On the right hand side we get

$$
P_{A_{1}^{2}\left(\Gamma, \alpha_{-}\right)}\left\{e^{-}(0, t)+\bar{t}\left(s_{-} e^{-}\right)(0, \bar{t})\right\}=\left(I+\mathcal{H}_{s_{-}}\right) e^{-}(0, t) .
$$

To evaluate the left hand side, using (1.10), we write

$$
\begin{aligned}
s(t) e^{+}(-1, t) & =s(0)\left(b e^{+}\right)(-1,0) \frac{k^{\alpha_{-}^{-1} \mu}(t)}{b(t) k^{\alpha_{-}^{-1} \mu}(0)}+g(t) \\
& =\frac{b^{\prime}(0)}{e^{-}(0,0)} \frac{k^{\alpha_{-}^{-1} \mu}(t)}{b(t) k^{\alpha_{-}^{-1} \mu}(0)}+g(t), \quad g \in A_{1}^{2}\left(\Gamma, \alpha_{-}^{-1}\right)
\end{aligned}
$$

Using (1.12), we get

$$
P_{A_{1}^{2}\left(\Gamma, \alpha_{-}\right)}\left\{\bar{t}\left(s e^{+}\right)(-1, \bar{t})\right\}=\frac{k^{\alpha_{-}}(t)}{e^{-}(0,0)}=\left(I+\mathcal{H}_{s_{-}}\right) e^{-}(0, t) .
$$

Thus,

$$
e^{-}(0, t) e^{-}(0,0)=\left(I+\mathcal{H}_{s_{-}}\right)^{-1} k^{\alpha_{-}} .
$$

In particular, $e^{-}(0,0)=K_{s_{-}}^{\alpha_{-}}(0)$, and (1.10) becomes the statement of the lemma.

Lemma 1.7. Assume that for some Jacobi matrix $J$ there exists a pair of unitary transforms

$$
\mathcal{F}^{ \pm}: l^{2}(\mathbb{Z}) \rightarrow L_{s_{ \pm}}^{2}, \quad\left(\mathcal{F}^{ \pm} J f\right)(t)=z(t)\left(\mathcal{F}^{ \pm} f\right)(t)
$$

determining each other by the relations

$$
s(t)\left(\mathcal{F}^{ \pm} f\right)(t)=\bar{t}\left(\mathcal{F}^{\mp} f\right)(\bar{t})+s_{\mp}(t)\left(\mathcal{F}^{\mp} f\right)(t),
$$

such that

$$
s \mathcal{F}^{ \pm}\left(l^{2}\left(\mathbb{Z}_{ \pm}\right)\right) \subset A_{1}^{2}\left(\Gamma, \alpha_{\mp}^{-1}\right) .
$$

As before, we put

$$
e^{+}(n, t)=\left(\mathcal{F}^{+} e_{n}\right)(t), \quad e^{-}(n, t)=\left(\mathcal{F}^{-} e_{-n-1}\right)(t)
$$

Then $e^{ \pm}(n, t)$ has at the origin zero (poles) of multiplicity $n, n>0(-n, n<0)$. Furthermore, $\mathcal{F}^{ \pm}\left(l^{2}\left(\mathbb{Z}_{ \pm}\right)\right) \supset H_{s_{ \pm}}^{2}\left(\alpha_{ \pm}\right)$, and, hence,

$$
e^{ \pm}(0,0) \geq K_{s_{ \pm}}^{\alpha_{ \pm}}(0)
$$

The equality in (1.15) takes place if and only if $e^{ \pm}(0, t)=K_{s_{ \pm}}^{\alpha_{ \pm}}(t)$.

Proof. Let us show that the annihilator of the linear space $A_{1}^{2}\left(\Gamma, \alpha^{+}\right) \subset L_{s_{+}}^{2}$ contains $\mathcal{F}^{+}\left\{l^{2}\left(\mathbb{Z}_{-}\right)\right\}$. For $f \in A_{1}^{2}\left(\Gamma, \alpha^{+}\right)$and $e^{+}(-n-1, t), n \geq 0$, we have

$$
\begin{aligned}
\left\langle f(t), e^{+}(-n-1, t)\right\rangle_{s_{+}} & =\frac{1}{2}\left\langle\left[\begin{array}{c}
f(t) \\
\bar{t} f(\bar{t})
\end{array}\right],\left[\begin{array}{cc}
1 & \overline{s_{+}(t)} \\
s_{+}(t) & 1
\end{array}\right]\left[\begin{array}{c}
e^{+}(-n-1, t) \\
\bar{t} e^{+}(-n-1, \bar{t})
\end{array}\right]\right\rangle \\
& =\left\langle f(t), e^{+}(-n-1, t)+\overline{t s_{+}(t)} e^{+}(-n-1, \bar{t})\right\rangle \\
& =\left\langle f(t), \bar{t}\left(s e^{-}\right)(n, \bar{t})\right\rangle .
\end{aligned}
$$


By (1.13) and (DCT), the last scalar product equals zero. Therefore,

$$
H_{s_{+}}^{2}\left(\alpha_{+}\right)=\operatorname{clos}_{L_{s_{+}}^{2}} A_{1}^{2}\left(\Gamma, \alpha^{+}\right) \subset\left\{\mathcal{F}^{+}\left(l^{2}\left(\mathbb{Z}_{-}\right)\right)\right\}^{\perp}=\mathcal{F}^{+}\left(l^{2}\left(\mathbb{Z}_{+}\right)\right) .
$$

Now, from the three-term recurrent relation

$$
z(t) s(t) e^{+}(n, t)=p_{n} s(t) e^{+}(n-1, t)+q_{n} s(t) e^{+}(n, t)+p_{n+1} s(t) e^{+}(n+1, t),
$$

and (1.13) it follows that $e^{+}(n, t), n>0$, has in the origin zero, at least of multiplicity $n$.

Since $K_{s_{+}}^{\alpha_{+}}(t) \in \mathcal{F}^{+}\left(l^{2}\left(\mathbb{Z}_{+}\right)\right)$, it possesses the decomposition

$$
K_{s_{+}}^{\alpha_{+}}(t)=\sum_{n \in \mathbb{Z}_{+}} a_{n} e^{+}(n, t) .
$$

Since $e^{+}(n, 0)=0, n>0$,

$$
a_{0}=\frac{K_{s_{+}}^{\alpha_{+}}(0)}{e^{+}(0,0)}
$$

in this decomposition. But,

$$
\left|a_{0}\right|^{2} \leq \sum\left|a_{n}\right|^{2}=\left\|K_{s_{+}}^{\alpha_{+}}(t)\right\|_{s_{+}}^{2}=1
$$

Thus, (1.15) and the lemma are proved.

Lemma $1.8[8]$. Let $f \in L^{\infty}\left(\alpha^{-2}\right)$. Then

$$
P_{A_{1}^{2}(\Gamma, \alpha)}\left\{\bar{t}\left(f b^{n} K^{\alpha \mu^{-n}}\right)(\bar{t})\right\} \rightarrow 0, \quad n \rightarrow+\infty,
$$

where $P_{A_{1}^{2}(\Gamma, \alpha)}$ is the orthogonal projection from $L_{d m \mid \mathbb{E}}^{2}$ onto $A_{1}^{2}(\Gamma, \alpha)$.

Proof. Let us denote by $\Delta^{\beta}(t)$ an extremal function of the problem

$$
\Delta^{\beta}(0)=\sup \left\{\phi(0): \phi \in H^{\infty}(\Gamma, \beta),\|\phi\| \leq 1\right\} .
$$

Using properties 1), 2) of a group of Widom type with (DCT), Theorem [5], and compactness of $\Gamma^{*}$, for any $\epsilon>0$, we can find a finite covering of $\Gamma^{*}$

$$
\Gamma^{*}=\bigcup_{j=1}^{l(\epsilon)}\left\{\beta: \operatorname{dist}\left(\beta, \beta_{j}\right) \leq \eta(\epsilon)\right\}
$$

such that

$$
2\left|1-\Delta^{\beta_{j}^{-1} \beta}(0) \frac{K^{\beta_{j}}(0)}{K^{\beta}(0)}\right| \leq \epsilon^{2}, \quad \operatorname{dist}\left(\beta, \beta_{j}\right) \leq \eta(\epsilon) .
$$

It means that

$$
\left\|\left(\Delta^{\beta_{j}^{-1} \beta} K^{\beta_{j}}\right)-K^{\beta}\right\|^{2} \leq 1+1-2 \Delta^{\beta_{j}^{-1} \beta}(0) \frac{K^{\beta_{j}}(0)}{K^{\beta}(0)} \leq \epsilon^{2}, \quad \operatorname{dist}\left(\beta, \beta_{j}\right) \leq \eta(\epsilon) .
$$


For fixed $\beta$ one can find $n_{0}$ such that

$$
\left\|P_{b^{n}} A_{1}^{2}\left(\Gamma, \alpha^{2} \beta^{-1} \mu^{-n}\right) \bar{t}\left(f K^{\beta}\right)(\bar{t})\right\| \leq \epsilon, \forall n>n_{0},
$$

Therefore, there exists $n_{0}$ such that

$$
\left\|P_{b^{n} A_{1}^{2}\left(\Gamma, \alpha^{2} \beta_{j}^{-1} \mu^{-n}\right)} \bar{t}\left(f K^{\beta_{j}}\right)(\bar{t})\right\| \leq \epsilon, \forall n>n_{0}, 1 \leq j \leq l(\epsilon) .
$$

Now, let $n>n_{0}=n_{0}(\epsilon)$ and let $\beta_{j}: \operatorname{dist}\left(\beta_{j}, \alpha \mu^{-n}\right) \leq \eta(\epsilon)$. For $h \in A_{1}^{2}(\Gamma, \alpha)$, we write $\left\langle\bar{t}\left(f b^{n} K^{\alpha \mu^{-n}}\right)(\bar{t}), h\right\rangle=\left\langle\bar{t}\left(b^{n} f\left[K^{\alpha \mu^{-n}}-\Delta^{\alpha \mu^{-n} \beta_{j}^{-1}} K^{\beta_{j}}\right]\right)(\bar{t}), h\right\rangle+\left\langle\bar{t}\left(b^{n} \Delta^{\alpha \mu^{-n} \beta_{j}^{-1}} f K^{\beta_{j}}\right)(\bar{t}), h\right\rangle$.

Then

$\left|\left\langle\bar{t}\left(b^{n} f\left[K^{\alpha \mu^{-n}}-\Delta^{\alpha \mu^{-n} \beta_{j}^{-1}} K^{\beta_{j}}\right]\right)(\bar{t}), h\right\rangle\right| \leq\|f\|\|h\|\left\|K^{\alpha \mu^{-n}}-\Delta^{\alpha \mu^{-n} \beta_{j}^{-1}} K^{\beta_{j}}\right\| \leq \epsilon\|f\|\|h\|$,

and

$$
\begin{aligned}
& \left|\left\langle\bar{t}\left(b^{n} \Delta^{\alpha \mu^{-n} \beta_{j}^{-1}} f K^{\beta_{j}}\right)(\bar{t}), h\right\rangle\right|=\left|\left\langle\bar{t}\left(f K^{\beta_{j}}\right)(\bar{t}), b^{n} \overline{\Delta^{\alpha \mu^{-n} \beta_{j}^{-1}}(\bar{t}} h\right\rangle\right| \\
& \leq\left\|P_{b^{n} A_{1}^{2}\left(\Gamma, \alpha^{2} \beta_{j}^{-1} \mu^{-n}\right)} \bar{t}\left(f K^{\beta_{j}}\right)(\bar{t})\right\|\|h\| \leq \epsilon\|h\| .
\end{aligned}
$$

Therefore,

$$
\left|\left\langle P_{A_{1}^{2}(\Gamma, \alpha)}\left\{\bar{t}\left(f b^{n} K^{\alpha \mu^{-n}}\right)(\bar{t})\right\}, h\right\rangle\right| \leq \epsilon(1+\|f\|)\|h\| .
$$

Putting $h=P_{A_{1}^{2}(\Gamma, \alpha)}\left\{\bar{t}\left(f b^{n} K^{\alpha \mu^{-n}}\right)(\bar{t})\right\}$, we get

$$
\left\|P_{A_{1}^{2}(\Gamma, \alpha)}\left\{\bar{t}\left(f b^{n} K^{\alpha \mu^{-n}}\right)(\bar{t})\right\}\right\| \leq \epsilon(1+\|f\|) .
$$

The lemma is proved.

Proposition 1.1. Assume that for some Jacobi matrix $J$ there exists a pair of unitary transforms

$$
\mathcal{F}^{ \pm}: l^{2}(\mathbb{Z}) \rightarrow L_{s_{ \pm}}^{2}, \quad\left(\mathcal{F}^{ \pm} J f\right)(t)=z(t)\left(\mathcal{F}^{ \pm} f\right)(t),
$$

determining each other by the relations

$$
s(t)\left(\mathcal{F}^{ \pm} f\right)(t)=\bar{t}\left(\mathcal{F}^{\mp} f\right)(\bar{t})+s_{\mp}(t)\left(\mathcal{F}^{\mp} f\right)(t),
$$

such that (1.13) holds. Then the following relations are equivalent:

$$
\begin{aligned}
& e^{ \pm}(n, t)=b^{n}(t) K^{\alpha_{ \pm} \mu^{-n}}+o(1) \quad \text { in } L_{s_{+}}^{2} ; \\
& \bar{t} p_{n}\left\{e^{+}(n, t) e^{+}(n-1, \bar{t})-e^{+}(n-1, t) e^{+}(n, \bar{t})\right\}=z^{\prime}(t) ; \\
& s(0) e^{+}(0,0)\left(b e^{-}\right)(-1,0)=b^{\prime}(0),
\end{aligned}
$$


where $\left\{e^{ \pm}(n, t)\right\}$ is defined by (1.14).

Proof. (1.18) $\Rightarrow(1.19)$. It follows from two remarks. First, the form on the left in (1.19) does not depend on $n$ ( it is the Wronskian of recurrence relation (0.7)). Second, the identity

$$
\bar{t} \frac{K^{\alpha}(0)}{K^{\alpha \mu}(0)}\left\{K^{\alpha}(t)\left(K^{\alpha \mu} / b\right)(\bar{t})-\left(K^{\alpha \mu} / b\right)(t) K^{\alpha}(\bar{t})\right\}=z^{\prime}(t)
$$

holds for any $\alpha \in \Gamma^{*}$.

$(1.19) \Rightarrow(1.20)$. Let us introduce the matrix

$$
\Phi(t)=\left[\begin{array}{ll}
e^{-}(-1, t) & -e^{-}(0, t) \\
-e^{+}(0, t) & e^{+}(-1, t)
\end{array}\right]
$$

Then (1.17) implies

$$
\bar{t} \Phi(\bar{t})=-S(t) \Phi(t) .
$$

In particular, with a help of (1.19), we get

$$
\begin{aligned}
s(t) & =-\bar{t} \frac{e^{+}(0, t) e^{+}(-1, \bar{t})-e^{+}(-1, t) e^{+}(0, \bar{t})}{e^{-}(-1, t) e^{+}(-1, t)-e^{-}(0, t) e^{+}(0, t)} \\
& =\frac{-z^{\prime}(t)}{p_{0}\left\{e^{-}(-1, t) e^{+}(-1, t)-e^{-}(0, t) e^{+}(0, t)\right\}} .
\end{aligned}
$$

Since $b(t) e^{ \pm}(-1, t)$ are holomorphic functions (in fact, of Smirnov class)

$$
s(0)=\frac{b^{\prime}(0)}{p_{0}\left(b e^{-}\right)(-1,0)\left(b e^{+}\right)(-1,0)} .
$$

Now, we only have to mention that $p_{0}\left(b e^{ \pm}\right)(-1,0)=e^{ \pm}(0,0)$. that

$(1.20) \Rightarrow(1.18)$. This is non-trivial part of the proposition. The main step is to prove

$$
\lim _{n \rightarrow+\infty} \frac{\left(b^{-n} e^{+}\right)(n, 0)}{K^{\alpha+\mu^{-n}}(0)}=1 .
$$

By Lemma (1.7) we have an estimate from below

$$
\begin{aligned}
\left(b^{-n} e^{+}\right)(n, 0) & \geq K_{s_{+} b^{2 n}}^{\alpha_{+}}(0) \geq\left\{\left(\epsilon+I+\mathcal{H}_{s_{+} b^{2 n}}\right)^{-1} k^{\alpha_{+} \mu^{-n}}\right\}^{1 / 2}(0) \\
& =\frac{1}{\sqrt{1+\epsilon}} K_{\frac{s_{+}}{1+\epsilon} b^{2 n}}^{\alpha_{+} \mu^{-n}}(0) .
\end{aligned}
$$

To get an estimate from above we use (1.20).

Let us note that due to the recurrence relation, the form

$$
p_{n}\left\{e^{+}(n-1, t) e^{-}(-n-1, t)-e^{+}(n, t) e^{-}(-n, t)\right\}
$$


also does not depend on $n$. Thus, a relation like (1.20) holds for all $n$ :

$$
\begin{aligned}
\left(b^{-n} e^{+}\right)(n, 0)\left(b^{n+1} e^{-}\right)(-n-1,0) & =p_{n}\left(b^{-n+1} e^{+}\right)(n-1,0)\left(b^{n+1} e^{-}\right)(-n-1,0) \\
& =e^{+}(0,0)\left(b e^{-}\right)(-1,0)=b^{\prime}(0) / s(0)
\end{aligned}
$$

Therefore,

$$
\begin{aligned}
\left(b^{-n} e^{+}\right)(n, 0) & =\frac{b^{\prime}(0)}{s(0)} \frac{1}{\left(b^{n+1} e^{-}\right)(-n-1,0)} \\
& \leq \frac{b^{\prime}(0)}{s(0)} \frac{1}{K_{s_{-} b^{-2 n-2}}^{\alpha_{-}(0)}} \\
& \leq \frac{b^{\prime}(0)}{s(0)} \frac{1}{\left\{\left(\epsilon+I+\mathcal{H}_{s_{-} b^{-2 n-2}}\right)^{-1} k^{\alpha_{-} \mu^{n+1}}\right\}^{1 / 2}(0)} \\
& =\frac{b^{\prime}(0)}{s(0)} \frac{\sqrt{1+\epsilon}}{K_{s_{\epsilon,-} b^{-2 n-2}}^{\alpha_{-} \mu^{n+1}}(0)},
\end{aligned}
$$

where $s_{\epsilon,-}:=s_{-} /(1+\epsilon)$.

With the function $s_{\epsilon,-}$, let us associate the functions $s_{\epsilon}, s_{\epsilon,+}$ and the character $\alpha_{\epsilon,+}$ (note, that $s_{\epsilon,+}$ is not $\frac{1}{1+\epsilon} s_{+}$, but $s_{\epsilon,+}=-\bar{s}_{\epsilon,-}\left(s_{\epsilon} / \bar{s}_{\epsilon}\right)$ ). It is important that $s_{\epsilon}(0)$ and $\alpha_{\epsilon,+}$ depend continuously on $\epsilon$.

By Lemma 1.6

$$
\frac{b^{\prime}(0)}{K_{s_{\epsilon,-} b^{-2 n-2}}^{\alpha_{-} \mu^{n+1}}(0)}=s_{\epsilon}(0) K_{s_{\epsilon,+} b^{2 n}}^{\alpha_{\epsilon,+} \mu^{-n}}(0) .
$$

Substituting (1.26) in (1.25), and combining the result with (1.24), we obtain

$$
\frac{1}{\sqrt{1+\epsilon}} K_{\frac{s_{+}}{1+\epsilon} b^{2 n}}^{\alpha_{+} \mu^{-n}}(0) \leq\left(b^{-n} e^{+}\right)(n, 0) \leq \sqrt{1+\epsilon} \frac{s_{\epsilon}(0)}{s(0)} K_{s_{\epsilon,+} b^{2 n}}^{\alpha_{\epsilon,+} \mu^{-n}}(0)
$$

Lemma 1.8 implies that for any $f \in L^{\infty}\left(\Gamma, \alpha_{+}^{-2}\right)$ with $\|f\|<1$ we have

$$
\lim _{n \rightarrow+\infty} \frac{K_{f b^{2 n}}^{\alpha_{+} \mu^{-n}}(0)}{K^{\alpha_{+} \mu^{-n}}(0)} \rightarrow 1
$$

Indeed,

$$
\begin{aligned}
& \left|k_{f b^{2 n}}^{\alpha_{+} \mu^{-n}}(0)-k^{\alpha_{+} \mu^{-n}}(0)\right|=\left|\left\langle\mathcal{H}_{f b^{2 n}} k^{\alpha_{+} \mu^{-n}},\left(I+\mathcal{H}_{f b^{2 n}}\right)^{-1} k^{\alpha_{+} \mu^{-n}}\right\rangle\right| \\
& =\left|\left\langle\bar{t}\left(f b^{n} k^{\alpha_{+} \mu^{-n}}\right)(\bar{t}), b^{n}\left(I+\mathcal{H}_{f b^{2 n}}\right)^{-1} k^{\alpha_{+} \mu^{-n}}\right\rangle\right| \\
& \leq\left\|P_{A_{1}^{2}\left(\Gamma, \alpha_{+}\right)}\left\{\bar{t}\left(f b^{n} k^{\alpha_{+} \mu^{-n}}\right)(\bar{t})\right\}\right\|\left\|b^{n}\left(I+\mathcal{H}_{f b^{2 n}}\right)^{-1} k^{\alpha_{+} \mu^{-n}}\right\| \\
& \leq \frac{1}{1-\|f\|}\left\|P_{A_{1}^{2}\left(\Gamma, \alpha_{+}\right)}\left\{\bar{t}\left(f b^{n} k^{\alpha_{+} \mu^{-n}}\right)(\bar{t})\right\}\right\|\left\|k^{\alpha_{+} \mu^{-n}}\right\| \rightarrow 0,
\end{aligned}
$$


as $n \rightarrow+\infty$.

Also, since $\alpha_{\epsilon,+}$ depends continuously on $\epsilon$ and $K^{\alpha_{+}}(0)$ is continuous on a compact group $\Gamma^{*}$, for any $\delta>0$ we can choose $\epsilon$ so small that

$$
\frac{K^{\alpha_{\epsilon,+} \mu^{-n}}(0)}{K^{\alpha_{+} \mu^{-n}}(0)} \leq 1+\delta, \quad \forall n
$$

Thus, returning to (1.27), we obtain

$$
\frac{1}{\sqrt{1+\epsilon}} \leq \lim _{n \rightarrow \infty} \inf \frac{\left(b^{-n} e^{+}\right)(n, 0)}{K^{\alpha_{+} \mu^{-n}}(0)} \leq \lim _{n \rightarrow \infty} \sup \frac{\left(b^{-n} e^{+}\right)(n, 0)}{K^{\alpha_{+} \mu^{-n}}(0)} \leq \sqrt{1+\epsilon} \frac{s_{\epsilon}(0)}{s(0)}(1+\delta) .
$$

Since $\epsilon$ and $\delta$ are arbitrary small, (1.23) is proved.

Now we are in a position to prove (1.18). Consider the norm of the difference

$$
\left\|e^{+}(n, t)-b^{n} K^{\alpha_{+} \mu^{-n}}\right\|_{s_{+}}^{2}=1+\left\|b^{n} K^{\alpha_{+} \mu^{-n}}\right\|_{s_{+}}^{2}-2\left\langle e^{+}(n, t), b^{n} K^{\alpha_{+} \mu^{-n}}\right\rangle_{s_{+}} .
$$

Since

$$
\left\|b^{n} K^{\alpha_{+} \mu^{-n}}\right\|_{s_{+}}^{2}=1+\left\langle b^{n} K^{\alpha_{+} \mu^{-n}}, \bar{t}\left(s_{+} b^{n} K^{\alpha_{+} \mu^{-n}}\right)(\bar{t})\right\rangle,
$$

using Lemma 1.8, we conclude that

$$
\left\|b^{n} K^{\alpha_{+} \mu^{-n}}\right\|_{s_{+}}^{2} \rightarrow 1, n \rightarrow+\infty
$$

Let us evaluate the scalar product

$$
\begin{aligned}
\left\langle e^{+}(n, t), b^{n} K^{\alpha_{+} \mu^{-n}}\right\rangle_{s_{+}} & =\left\langle s e^{-}(-n-1, t), \bar{t}\left(b^{n} K^{\alpha_{+} \mu^{-n}}\right)(\bar{t})\right\rangle \\
& =\left\langle s e^{-}(-n-1, t), b^{-n} b^{-1} K^{\alpha_{+}^{-1} \mu^{n+1}}\right\rangle \\
& =\frac{s(0)\left(b^{n+1} e^{-}\right)(-n-1,0)}{K^{\alpha_{+}^{-1} \mu^{n+1}}(0)} \\
& =\frac{K^{\alpha_{+} \mu^{-n}}(0)}{\left(b^{-n} e^{+}\right)(n, 0)} \rightarrow 1, n \rightarrow+\infty .
\end{aligned}
$$

The proposition is proved.

The following theorem shows that an arbitrary function $s_{+}$, possessing $(1.1),(1.2)$, is the reflection coefficient of a Jacobi matrix of Szegö class.

Theorem 1.1. Let a function $s_{+} \in L^{\infty}\left(\Gamma, \alpha_{+}^{-2}\right),\left\|s_{+}\right\| \leq 1, \overline{s_{+}(\bar{t})}=s_{+}(t)$, be such that that $\log \left(1-\left|s_{+}\right|^{2}\right) \in L^{1}$. Let an outer function $s, s(0)>0$, and $s_{-}$be associated to $s_{+}$by the relations

$$
|s|^{2}=1-\left|s_{+}\right|^{2}, \quad s_{-}=-\bar{s}_{+} s / \bar{s}
$$

Then the system of functions

$$
e^{+}(n, t)=b^{n} K_{s_{+} b^{2 n}}^{\alpha_{+} \mu^{-n}}
$$


forms an orthonormal basis in $L_{s_{+}}^{2}$. The dual system, defined by

$$
s(t) e^{-}(n, t)=\bar{t} e^{+}(-n-1, \bar{t})+s_{+}(t) e^{+}(-n-1, t),
$$

forms an orthonormal basis in $L_{s_{-}}^{2}$. The subspaces of $L_{s_{ \pm}}^{2}$, that formed by functions with vanishing negative Fourier coefficients with respect to these basses, are spaces of holomorphic character-automorphic forms, moreover,

$$
s f^{ \pm} \in A_{1}^{2}\left(\Gamma, \alpha_{\mp}^{-1}\right) \text { if } f^{ \pm} \in \operatorname{clos}_{L_{s_{ \pm}}^{2}} \operatorname{span}\left\{e^{ \pm}(n, t): n \geq 0\right\} .
$$

Further,

$$
e^{ \pm}(n, t)=b^{n} K^{\alpha_{ \pm} \mu^{-n}}+o(1) \text { in } L_{s_{ \pm}}^{2},
$$

and with respect to these basses the operator multiplication by $z(t)$ is a Jacobi matrix $J$ of Szegö class.

Proof. All statements, besides the last one, only summaries results of Lemmas 1.4, 1.5 and Proposition 1.1. To prove that $J$ is of Szegö class we evaluate its spectral density $\rho(x)$.

Using the definition of the resolvent matrix-function, we get

$$
R(z)=\left[\begin{array}{cc}
\left\langle(z(t)-z)^{-1} e^{+}(-1, t), e^{+}(-1, t)\right\rangle_{s_{+}} & \left\langle(z(t)-z)^{-1} e^{+}(0, t), e^{+}(-1, t)\right\rangle_{s_{+}} \\
\left\langle(z(t)-z)^{-1} e^{+}(-1, t), e^{+}(0, t)\right\rangle_{s_{+}} & \left\langle(z(t)-z)^{-1} e^{+}(0, t), e^{+}(0, t)\right\rangle_{s_{+}}
\end{array}\right] .
$$

Note, that if $f^{ \pm} \in L_{s_{ \pm}}^{2}$ are related by $s(t) f^{-}(t)=\bar{t} f^{+}(\bar{t})+s_{+}(t) f^{+}(t)$ then

$$
\left(\frac{f^{+}(t)}{z(t)-z}\right)^{-}=\frac{f^{-}(t)}{z(t)-z}
$$

Therefore, using Lemma 1.3, we have

$$
R(z)=\frac{1}{2} \int_{\mathbb{E}}\left[\begin{array}{cc}
e^{+}(-1, t) & e^{+}(0, t) \\
e^{-}(0, t) & e^{-}(-1, t)
\end{array}\right]^{*}\left[\begin{array}{cc}
e^{+}(-1, t) & e^{+}(0, t) \\
e^{-}(0, t) & e^{-}(-1, t)
\end{array}\right] \frac{|s(t)|^{2} d m}{z(t)-z},
$$

and, substituting $s(t)$ from (1.22), we obtain

$$
\begin{aligned}
R(z) & =\frac{1}{2} \int_{\mathbb{E}} \frac{\left[\begin{array}{cc}
e^{+}(-1, t) & e^{+}(0, t) \\
e^{-}(0, t) & e^{-}(-1, t)
\end{array}\right]^{*}\left[\begin{array}{cc}
e^{+}(-1, t) & e^{+}(0, t) \\
e^{-}(0, t) & e^{-}(-1, t)
\end{array}\right]}{p_{0}^{2}\left|e^{-}(-1, t) e^{+}(-1, t)-e^{-}(0, t) e^{+}(0, t)\right|^{2}} \frac{\left|z^{\prime}(t)\right|^{2} d m}{z(t)-z} \\
& =\frac{1}{2} \int_{\mathbb{E}} \frac{\tilde{\Phi}^{-1 *}(t) \tilde{\Phi}^{-1}(t)}{z(t)-z} \frac{\left|z^{\prime}(t)\right|^{2}|d t|}{2 \pi p_{0}^{2}}
\end{aligned}
$$

where

$$
\tilde{\Phi}(t)=\left[\begin{array}{ll}
e^{-}(-1, t) & -e^{+}(0, t) \\
-e^{-}(0, t) & e^{+}(-1, t)
\end{array}\right] .
$$

Thus,

$$
2 \pi p_{0}^{2} \rho(z(t))=\tilde{\Phi}^{-1 *}(t) \tilde{\Phi}^{-1}(t)\left|z^{\prime}(t)\right|
$$

and

$$
\operatorname{det}\left\{2 \pi p_{0} \rho(z(t))\right\}=\frac{\left|z^{\prime}(t)\right|^{2}}{p_{0}^{2}|\operatorname{det} \tilde{\Phi}(t)|^{2}}=|s(t)|^{2} .
$$

The theorem is proved.

Let us note, by the way, that $\Phi(t)$ (see (1.21)) and $\tilde{\Phi}(t)$ are related by $\tilde{\Phi}(\bar{t})=\Phi^{*}(t)$ and, besides (1.28),

$$
2 \pi p_{0}^{2} \rho(z(t))=\Phi^{-1}(t) \Phi^{-1 *}(t)\left|z^{\prime}(t)\right| .
$$




\section{FROM SPECTRAL DATA TO SCATTERING DATA}

We start this section with the remark that the spectral measure $d \sigma$ determines a Jacobi matrix uniquely, but it is not an arbitrary $2 \times 2$ matrix-measure, or, say, a real-valued (all entries are real) $2 \times 2$ matrix-measure.

Indeed, one can represent $J$ as a two dimensional perturbation of an orthogonal sum of a pair of one-sided Jacobi matrices, i.e.:

$$
J=\left[\begin{array}{cc}
J_{-} & 0 \\
0 & J_{+}
\end{array}\right]+p_{0}\left\langle, e_{-1}\right\rangle e_{0}+p_{0}\left\langle, e_{0}\right\rangle e_{-1}
$$

where $J_{ \pm}=P_{l^{2}\left(\mathbb{Z}_{ \pm}\right)} J \mid l^{2}\left(\mathbb{Z}_{ \pm}\right)$. This formula implies that

$$
R(z)=\left[\begin{array}{cc}
r_{-}^{-1}(z) & p_{0} \\
p_{0} & r_{+}^{-1}(z)
\end{array}\right]^{-1}
$$

where

$$
\begin{aligned}
& r_{-}(z)=r\left(z, J_{-}\right)=\left\langle\left(J_{-}-z\right)^{-1} e_{-1}, e_{-1}\right\rangle=\int \frac{d \sigma_{-}(x)}{x-z} \\
& r_{+}(z)=r\left(z, J_{+}\right)=\left\langle\left(J_{+}-z\right)^{-1} e_{0}, e_{0}\right\rangle=\int \frac{d \sigma_{+}(x)}{x-z}
\end{aligned}
$$

Thus, the real-valued matrix-measure $d \sigma$ is determined by two scalar measures $d \sigma_{ \pm}$(with the normalization $\int d \sigma_{ \pm}=1$ ) and a constant $p_{0}$.

In what follows $\hat{f}(x) \in L_{d \sigma}^{2}$ denotes the image of $f \in l^{2}(\mathbb{Z})$ in the spectral representation. Recall that

$$
\hat{e}_{-1}=\left[\begin{array}{l}
1 \\
0
\end{array}\right], \quad \hat{e}_{0}=\left[\begin{array}{l}
0 \\
1
\end{array}\right]
$$

and

$$
(\widehat{J f})(x)=x \hat{f}(x) .
$$

Let $\left\{P_{n}^{ \pm}(z)\right\}$ be the orthonormal polynomials with respect to the (scalar) measure $d \sigma_{ \pm}$ and

$$
Q_{n}^{ \pm}(z):=\int \frac{P_{n}^{ \pm}(x)-P_{n}^{ \pm}(z)}{x-z} d \sigma_{ \pm}(x)
$$

(so-called polynomials of the second kind). In these terms

$$
\begin{aligned}
\hat{e}_{n}(x) & =\left[\begin{array}{c}
-p_{0} Q_{n}^{+}(x) \\
P_{n}^{+}(x)
\end{array}\right], \quad n \geq 0 \\
\hat{e}_{-n-1}(x) & =\left[\begin{array}{c}
P_{n}^{-}(x) \\
-p_{0} Q_{n}^{-}(x)
\end{array}\right], \quad n \geq 0 .
\end{aligned}
$$

Now, we prove Theorem 0.5 . 
Proof of Theorem 0.5, the uniqueness part. The function $e^{ \pm}(0, \zeta) / e^{ \pm}(-1, \zeta)$ is $\Gamma$-automorphic, thus it defines a meromorphic function in $\overline{\mathbb{C}} \backslash E$,

$$
\tilde{r}_{ \pm}(z(\zeta)):=-\frac{e^{ \pm}(0, \zeta)}{p_{0} e^{ \pm}(-1, \zeta)}
$$

The recurrence relations implies that $\tilde{r}_{ \pm}(z)$ possesses the same decomposition into a continued fraction as $r_{ \pm}(z)$. Therefore,

$$
r_{ \pm}(z(\zeta))=-\frac{e^{ \pm}(0, \zeta)}{p_{0} e^{ \pm}(-1, \zeta)}
$$

By Proposition 1.1 the asymptotic (1.18) implies the identity (1.19). Using this identity, we get $(t \in \mathbb{T})$

$$
r_{ \pm}(z(t))-\overline{r_{ \pm}(z(t))}=-p_{0} \frac{e^{ \pm}(0, t) \overline{e^{ \pm}(-1, t)}-e^{ \pm}(-1, t) \overline{e^{ \pm}(0, t)}}{\left|p_{0} e^{ \pm}(-1, t)\right|^{2}}=\frac{-t z^{\prime}(t)}{\left|p_{0} e^{ \pm}(-1, t)\right|^{2}}
$$

This means that an outer part of the function $e^{ \pm}(-1, \zeta)$ is determined uniquely. But then (2.3) means that an outer part of $e^{ \pm}(0, \zeta)$ is determined uniquely, and since $b(\zeta) e^{ \pm}(-1, \zeta)$ and $e^{ \pm}(0, \zeta)$ are of Smirnov class, these functions are determined up to a common inner factor $\Delta_{ \pm}(\zeta)$, i.e.,

$$
e^{ \pm}(0, \zeta)=\Delta_{ \pm}(\zeta) \tilde{e}^{ \pm}(0, \zeta) \text { and } e^{ \pm}(-1, \zeta)=\Delta_{ \pm}(\zeta) \tilde{e}^{ \pm}(-1, \zeta),
$$

where the inner parts of $\tilde{e}^{ \pm}(0, \zeta), \tilde{e}^{ \pm}(-1, \zeta)$ are relatively prime.

To show that $\Delta_{ \pm}(\zeta)=1$ we use $(0.23),(0.24)$. Since

$$
\begin{aligned}
s(t) e^{\mp}(0, t) & =\bar{t} e^{ \pm}(-1, \bar{t})+s_{ \pm}(t) e^{ \pm}(-1, t), \\
s(t) e^{\mp}(-1, t) & =\bar{t} e^{ \pm}(0, \bar{t})+s_{ \pm}(t) e^{ \pm}(0, t),
\end{aligned}
$$

we have

$$
s(t)\left\{e^{\mp}(-1, t) e^{ \pm}(-1, t)-e^{\mp}(0, t) e^{ \pm}(0, t)\right\}=\bar{t}\left\{e^{ \pm}(0, \bar{t}) e^{ \pm}(-1, t)-e^{ \pm}(-1, \bar{t}) e^{ \pm}(0, t)\right\} .
$$

Substituting (2.4) and using the symmetry

$$
\overline{\tilde{e}^{ \pm}(0, \bar{t})}=\tilde{e}^{ \pm}(0, t), \quad \overline{\tilde{e}^{ \pm}(-1, \bar{t})}=\tilde{e}^{ \pm}(-1, t),
$$

we obtain

$$
\begin{aligned}
& s(t) b^{2}(t)\left\{e^{\mp}(-1, t) \tilde{e}^{ \pm}(-1, t)-e^{\mp}(0, t) \tilde{e}^{ \pm}(0, t)\right\} \\
& \quad=\bar{t} \Delta_{ \pm}(\bar{t}) b^{2}(t)\left\{\overline{\tilde{e}^{ \pm}(0, t)} \tilde{e}^{ \pm}(-1, t)-\overline{\tilde{e}^{ \pm}(-1, t)} \tilde{e}^{ \pm}(0, t)\right\} \\
& \quad=-b^{2}(t) z^{\prime}(t)\left\{p_{0} \overline{\Delta_{ \pm}(\bar{t})}\right\}^{-1} .
\end{aligned}
$$

Since the first expression here is a function of Smirnov class and $b^{2} z^{\prime}$ is an outer function we conclude that $\Delta_{ \pm}(t)$ is a constant. 
Since

$$
\bar{t} \Phi(\bar{t})=-S(t) \Phi(t)
$$

with $\Phi(t)$ defined by (1.21), $S(t)$ is also determined in a unique way. At last, by the recurrence relations we get the same conclusion with respect to all functions $\left\{e^{ \pm}(n, \zeta)\right\}$, not only for $n=-1,0$.

Proof of Theorem 0.5, the existence part. The key instrument is the following theorem [9]: if $r(z)$ is a meromorphic function in $\overline{\mathbb{C}} \backslash E$ such that $\operatorname{Im} r(z) / \operatorname{Im} z \geq 0$ and poles of $r(z(\zeta))$ satisfies the Blaschke condition, then $r(z(\zeta))$ is a function of bounded characteristic in $\mathbb{D}$ without a singular component in the multiplicative representation.

Let us show that poles of $r_{ \pm}(z(\zeta))$ satisfies the Blaschke condition. Diagonal entries $R_{-1,-1}(z)$ and $R_{0,0}(z)$ of the resolvent matrix-function $R(z)$ are holomorphic in $\overline{\mathbb{C}} \backslash E$. By the theorem mentioned above they are functions of bounded characteristic. In force of $(2.1)$,

$$
\begin{aligned}
-1 / R_{-1,-1}(z) & =-1 / r_{-}(z)+p_{0}^{2} r_{+}(z), \\
-1 / R_{0,0}(z) & =-1 / r_{+}(z)+p_{0}^{2} r_{-}(z) .
\end{aligned}
$$

This means that poles of $r_{ \pm}$are subsets of poles of $1 / R_{-1,-1}$ and $1 / R_{0,0}$. Thus $r_{ \pm}(z(\zeta))$ are functions of bounded characteristic.

Now, let us use the Szegö condition $\log \operatorname{det} \operatorname{Im} R(z(t)) \in L^{1}$. Since

$$
\operatorname{det} \operatorname{Im} R^{-1}(z(t))=\left|\operatorname{det} R^{-1}(z(t))\right|^{2} \operatorname{det} \operatorname{Im} R(z(t))
$$

using again (2.1), we have

$$
\log \operatorname{Im} r_{-}^{-1}(z(t))+\log \operatorname{Im} r_{+}^{-1}(z(t))=\log \operatorname{det} \operatorname{Im} R^{-1}(z(t)) \in L^{1} .
$$

Therefore, each of the functions $\log \operatorname{Im} r_{ \pm}(z(t))$ belongs to $L^{1}$. Thus we can represent $r_{ \pm}(z)$ (uniquely) in the form

$$
r_{ \pm}(z(\zeta))=-\frac{e^{ \pm}(0, \zeta)}{p_{0} e^{ \pm}(-1, \zeta)}
$$

where $e^{ \pm}(0, \zeta)$ and $b(\zeta) e^{ \pm}(-1, \zeta)$ are functions of Smirnov class with coprime inner parts (in fact, they are Blaschke products) such that

$$
\bar{t} p_{0}\left\{e^{ \pm}(0, t) e^{ \pm}(-1, \bar{t})-e^{ \pm}(-1, t) e^{ \pm}(0, \bar{t})\right\}=z^{\prime}(t)
$$

and $e^{ \pm}(0,0)>0,\left(b e^{ \pm}\right)(-1,0)>0$. Note that

$$
p_{0}=\frac{e^{ \pm}(0,0)}{\left(b e^{ \pm}\right)(-1,0)} .
$$

As soon as the functions $e^{ \pm}(0, \zeta)$ and $e^{ \pm}(-1, \zeta)$ have been constructed we are able to introduce $S(t)$ and $\mathcal{F}^{ \pm}$in their terms. 
First, let us write down an expression for the resolvent matrix-function:

$$
\begin{aligned}
R(z(\zeta)) & =\left[\begin{array}{cc}
-p_{0} \frac{e^{-}(-1, \zeta)}{e^{-}(0, \zeta)} & p_{0} \\
p_{0} & -p_{0} \frac{e^{+}(-1, \zeta)}{e^{+}(0, \zeta)}
\end{array}\right]^{-1} \\
& =-\left(p_{0} \Phi\right)^{-1} \Psi=-\tilde{\Psi}\left(p_{0} \tilde{\Phi}\right)^{-1}
\end{aligned}
$$

where $\Phi$ and $\tilde{\Phi}$ are as in (1.21) and (1.28) respectively, and

$$
\Psi(\zeta)=\tilde{\Psi}(\zeta)=\left[\begin{array}{cc}
e^{-}(0, \zeta) & 0 \\
0 & e^{+}(0, \zeta)
\end{array}\right] .
$$

Therefore,

$$
p_{0}^{2}\left\{R(z(t))-R^{*}(z(t))\right\}=-t z^{\prime}(t) \Phi^{-1}(t) \Phi^{-1 *}(t)=-t z^{\prime}(t) \tilde{\Phi}^{-1 *}(t) \tilde{\Phi}^{-1}(t),
$$

since (see (2.7))

$$
p_{0}\left\{\Psi \Phi^{*}-\Phi \Psi^{*}\right\}=p_{0}\left\{\tilde{\Phi}^{*} \tilde{\Psi}-\tilde{\Psi}^{*} \tilde{\Phi}\right\}=t z^{\prime} .
$$

From $(2.9)$ and $\tilde{\Phi}^{*}(t)=\Phi(\bar{t})$ we get immediately that the matrix-function $S(t)$ defined by (2.6) is unitary-valued. Let us show that its element $s(\zeta)$ is an outer function. In fact, we have to show that the function $b^{2}(\zeta) \operatorname{det} \Phi(\zeta)$ is an outer function (see (1.22)). To this end let us use the representation for the diagonal entries of $R(z)$ (see (2.8))

$$
\begin{aligned}
R_{-1,-1}(z(\zeta)) & =-\frac{e^{+}(-1, \zeta) e^{-}(0, \zeta)}{p_{0} \operatorname{det} \Phi(\zeta)}, \\
R_{0,0}(z(\zeta)) & =-\frac{e^{-(-1, \zeta) e^{+}(0, \zeta)}}{p_{0} \operatorname{det} \Phi(\zeta)} .
\end{aligned}
$$

Let $\Delta$ be an inner part of $b^{2}(\zeta) \operatorname{det} \Phi(\zeta)$. Since $R_{0,0}(z(\zeta))$ is of Smirnov class, $\Delta$ is a divisor of $e^{-}(-1, \zeta) e^{+}(0, \zeta)$. If $\Delta$ is not trivial, then it has a non-trivial divisor $\Delta_{1}$ that is a divisor of one of these functions, say, $e^{-}(-1, \zeta)$. Since $e^{-}(-1, \zeta)$ and $e^{-}(0, \zeta)$ are coprime (and $\Delta_{1}$ is a divisor of $\left.b^{2}(\zeta) \operatorname{det} \Phi(\zeta)\right)$, the $\Delta_{1}$ is a divisor of $e^{+}(0, \zeta)$, and, therefore, it is not a divisor of $e^{+}(-1, \zeta)$. Thus, $\Delta_{1}$ is not a divisor of the product $e^{+}(-1, \zeta) e^{-}(0, \zeta)$. But this means that $R_{-1,-1}(z(\zeta))$ is not of Smirnov class. We arrive to a contradiction, hence $\Delta$ is a constant.

We define $\mathcal{F}^{ \pm}$by the formulas

$$
\begin{aligned}
& \left(\mathcal{F}^{+} f\right)(t)=\left[e^{+}(-1, t) \quad e^{+}(0, t)\right] \hat{f}(z(t)), \\
& \left(\mathcal{F}^{-} f\right)(t)=\left[e^{-}(0, t) \quad e^{-}(-1, t)\right] \hat{f}(z(t)) .
\end{aligned}
$$

Evidently, $\left(\mathcal{F}^{ \pm} J f\right)(t)=z(t)\left(\mathcal{F}^{ \pm} f\right)(t)$ and by $(2.6),(0.23)$ are fulfilled. Using the formula for the spectral density $\rho(x)=\frac{1}{\pi} \operatorname{Im} R(x)$ and (2.9), we have

$$
\int_{E} \hat{f}^{*}(x) \rho(x) d x \hat{f}(x)=\frac{1}{2} \int_{\mathbb{E}} \hat{f}^{*}(z(t))\left(p_{0} \tilde{\Phi}\right)^{*-1}\left(p_{0} \tilde{\Phi}\right)^{-1} \hat{f}(z(t))\left|z^{\prime}(t)\right|^{2} d m(t) .
$$


Since

$$
\tilde{\Phi}^{-1}(t)=\frac{1}{\operatorname{det} \tilde{\Phi}(t)}\left[\begin{array}{cc}
e^{+}(-1, t) & e^{+}(0, t) \\
e^{-}(0, t) & e^{-}(-1, t)
\end{array}\right]
$$

we obtain

$$
\begin{aligned}
\|f\|^{2}=\|\hat{f}\|_{L_{d \sigma}^{2}}^{2} & =\frac{1}{2}\left\{\left\|s \mathcal{F}^{+} f\right\|^{2}+\left\|s \mathcal{F}^{-} f\right\|^{2}\right\} \\
& =\left\|\mathcal{F}^{+} f\right\|_{s_{+}}^{2}=\left\|\mathcal{F}^{-} f\right\|_{s_{-}}^{2} .
\end{aligned}
$$

Thus $\mathcal{F}^{+}$is an isometry, and since this map is invertible,

$$
\left[\begin{array}{c}
\hat{f}_{-1}(z(t)) \\
\hat{f}_{0}(z(t))
\end{array}\right]=-\frac{p_{0}}{z^{\prime}(t)}\left[\begin{array}{cc}
\bar{t} e^{+}(0, \bar{t}) & -e^{+}(0, t) \\
-\bar{t} e^{+}(-1, \bar{t}) & e^{+}(-1, t)
\end{array}\right]\left[\begin{array}{c}
g(t) \\
\bar{t} g(\bar{t})
\end{array}\right],
$$

where $g=\mathcal{F}^{+} f$, it is a unitary map.

Further, using (2.2), for $n \geq 0$ we have

$$
e^{+}(n, \zeta)=\left[e^{+}(-1, \zeta) \quad e^{+}(0, \zeta)\right]\left[\begin{array}{c}
-p_{0} Q_{n}^{+}(z(\zeta)) \\
P_{n}^{+}(z(\zeta))
\end{array}\right] .
$$

Due to the well known properties of orthogonal polynomials these functions have no singularity at the origin and hence they are functions of Smirnov class. This easily implies (0.24).

At last, our maps possess properties (1.19) (or (1.20)), in force of Proposition 1.1, (0.25) holds. The theorem is proved.

Theorem 2.1. Let $s_{+} \in L^{\infty}\left(\Gamma, \alpha_{+}^{-2}\right),\left\|s_{+}\right\| \leq 1, \overline{s_{+}(\bar{t})}=s_{+}(t)$, satisfy $\log \left(1-\left|s_{+}\right|^{2}\right) \in L^{1}$. Then the reflection coefficient $s_{+}$determines a Jacobi matrix of Szegö class in a unique way if and only if

$$
s(0) K_{s_{ \pm}}^{\alpha_{ \pm}}(0) K_{s_{\mp} b^{-2}}^{\alpha_{\mp} \mu}(0)=b^{\prime}(0) .
$$

Proof. Assume on the contrary that

$$
s(0) K_{s_{+}}^{\alpha_{+}}(0) K_{s_{-} b^{-2}}^{\alpha_{-} \mu}(0) \neq b^{\prime}(0) .
$$

We construct two Jacobi matrices. First, we consider the basis

$$
e^{+}(n, t)=b^{n}(t) K_{s_{+} b^{2 n}}^{\alpha_{+} \mu^{-n}}(t)
$$

and by $J$ we denote the operator multiplication by $z(t)$ in $L_{s_{+}}^{2}$ with respect to this basis (Lemma 1.4). Then, starting with the basis $\left\{b^{n}(t) K_{s_{-} b^{2 n}}^{\alpha_{-} \mu^{-n}}(t)\right\}$ in $L_{s_{-}}^{2}$, we introduce the basis

$$
s(t) \tilde{e}^{+}(-n-1, t)=\bar{t}\left(b^{n} K_{s_{-} b^{2 n}}^{\alpha_{-} \mu^{-n}}\right)(\bar{t})+s_{-}(t)\left(b^{n} K_{s_{-} b^{2 n}}^{\alpha_{-} \mu^{-n}}\right)(t) .
$$

By $\tilde{J}$ we denote the operator multiplication by $z(t)$ in $L_{s_{+}}^{2}$ with respect to $\left\{\tilde{e}^{+}(n, t)\right\}$. By Lemma 1.5,

$$
s(0) \tilde{e}^{+}(0,0) K_{s_{-} b^{-2}}^{\alpha_{-} \mu}(0)=b^{\prime}(0)
$$


Thus (see $(2.12)), \tilde{e}^{+}(0,0) \neq e^{+}(0,0)$. Due to the uniqueness part of Theorem $0.5, \tilde{J} \neq J$. The "only if" part is proved.

Now, let (2.11) holds, and let $J$ be a Jacobi matrix of Szegö class and $\mathcal{F}^{ \pm}$be its representations in $L_{s_{ \pm}}^{2}$. By Lemma 1.7,

$$
K_{s_{ \pm}}^{\alpha_{ \pm}}(0) \leq e^{ \pm}(0,0)=\frac{b^{\prime}(0)}{s(0)} \frac{1}{\left(b e^{\mp}\right)(-1,0)} \leq \frac{b^{\prime}(0)}{s(0)} \frac{1}{K_{s_{\mp} b^{-2}}^{\alpha_{\mp} \mu}(0)}
$$

Then (2.11) implies that, in fact, $e^{ \pm}(0,0)=K_{s_{ \pm}}^{\alpha_{ \pm}}(0)$ and $\left(b e^{\mp}\right)(-1,0)=K_{s_{\mp} b^{-2}}^{\alpha_{\mp} \mu}(0)$, thus, due to a conclusion of Lemma 1.7,

$$
e^{ \pm}(0, t)=K_{s_{ \pm}}^{\alpha_{ \pm}}(t), \quad e^{\mp}(-1, t)=b^{-1}(t) K_{s_{\mp} b^{-2}}^{\alpha_{\mp} \mu}(t) .
$$

Recall that these functions determine the functions $r_{ \pm}(z)$ and the coefficient $p_{0}$ (see (2.3)), and they, in their turn, determine $J$. The theorem is proved.

Corollary 2.1. Let $J$ be a Jacobi matrix of Szegö class with a homogeneous spectrum E. Let $\rho(x)$ be the density of its spectral measure and $S(t)$ be its scattering matrix-function. If

$$
\int_{E} \rho^{-1}(x) d x<\infty
$$

then there is no other Jacobi matrix of Szegö class with the same scattering matrix-function $S(t)$.

Proof. By virtue of (1.29), (2.15) is equivalent to

$$
\int_{\mathbb{E}} \tilde{\Phi}(t) \tilde{\Phi}^{*}(t) d m<\infty
$$

that is $e^{ \pm}(0, t)$ and $e^{ \pm}(-1, t)$ belong to $L_{d m \mid \mathbb{E}}^{2}$. Then words by words repetition of arguments in the proof of Lemma 1.6 gives us

$$
\begin{aligned}
\left(I+\mathcal{H}_{s_{ \pm}}\right) e^{ \pm}(0, t) e^{ \pm}(0,0) & =k^{\alpha_{ \pm}}(t), \\
\left(I+\mathcal{H}_{s_{ \pm} b^{-2}}\right)\left(b e^{ \pm}\right)(-1, t)\left(b e^{ \pm}\right)(-1,0) & =k^{\alpha_{ \pm} \mu}(t) .
\end{aligned}
$$

Thus, $e^{ \pm}(0,0)=K_{s_{ \pm}}^{\alpha_{ \pm}}(0)$ and $\left(b e^{ \pm}\right)(-1,0)=K_{s_{ \pm} b^{-2}}^{\alpha_{ \pm} \mu}(0)$. Since, generally,

$$
s(0) e^{ \pm}(0,0)\left(b e^{ \pm}\right)(-1,0)=b^{\prime}(0)
$$

(2.11) holds, the corollary is proved.

To finish this section we give an example of a scattering matrix-function, which does not determine a Jacobi matrix of Szegö class. Moreover, in this example, the associated operators $\left(I+\mathcal{H}_{s_{ \pm}}\right)$are invertible. 
Example. Let $v_{ \pm} \in H^{\infty}(\Gamma),\left\|v_{ \pm}\right\|<1, \overline{v_{ \pm}(\bar{t})}=v(t), v_{ \pm}(0)=0$. Define outer functions $u_{ \pm}, u_{ \pm}(0)>0$, by

$$
\left|u_{ \pm}\right|^{2}+\left|v_{ \pm}\right|^{2}=1
$$

Then, we put

$$
s_{ \pm}^{0}=-\bar{v}_{ \pm} u_{ \pm} / \bar{u}_{ \pm}
$$

At last,

$$
S(t)=\left[\begin{array}{cc}
s_{-} & s \\
s & s_{+}
\end{array}\right]=\left[\begin{array}{cc}
s_{-}^{0} & 0 \\
0 & s_{+}^{0}
\end{array}\right]+\left[\begin{array}{cc}
u_{-} & 0 \\
0 & u_{+}
\end{array}\right] \mathcal{E}\left\{I-\left[\begin{array}{cc}
v_{-} & 0 \\
0 & v_{+}
\end{array}\right] \mathcal{E}\right\}^{-1}\left[\begin{array}{cc}
u_{-} & 0 \\
0 & u_{+}
\end{array}\right]
$$

where

$$
\mathcal{E}=\left[\begin{array}{ll}
\frac{1+\Delta}{2} & \frac{1-\Delta}{2} \\
\frac{1-\Delta}{2} & \frac{1+\Delta}{2}
\end{array}\right]
$$

and $\Delta$ is an inner function from $H^{\infty}(\Gamma), \overline{\Delta(\bar{t})}=\Delta(t)$.

In this case $\mathcal{H}_{s_{ \pm}}=\mathcal{H}_{s_{ \pm}^{0}}$, since their symbols differ by functions from $H^{\infty}\left(\Gamma, \alpha_{ \pm}^{-2}\right)$, and therefore $\left(I+\mathcal{H}_{s_{ \pm}}\right)$are invertible. From the other hand, the coefficient $s$ is of the form

$$
s=\frac{u_{+} u_{-}(1-\Delta) / 2}{1-\left(v_{+}+v_{-}\right)(1+\Delta) / 2+v_{+} v_{-} \Delta}
$$

and because of the factor $(1-\Delta) / 2,1 / s$ does not belong to $H^{\infty}\left(\Gamma, \alpha_{+} \alpha_{-}\right)$.

The simplest choice of parameters:

$$
E=[-2,2], \quad v_{ \pm}(t)=a_{ \pm} t, 0<a_{ \pm}<1,
$$

$\Delta(t)$ is a Blaschke product, $\operatorname{deg} \Delta>1$, gives us an example where $\tilde{e}^{+}(-1, t)$, defined by (2.14), does not belong to $L^{2}$, at the same time $e^{+}(-1, t)$, defined by $(2.13)$, belongs to $L^{2}$.

\section{The Hilbert transform And $A_{2}$ CONDition}

By $\mathfrak{H}$ we denote the transform

$$
(\mathfrak{H} g)(z)=\int_{E} \frac{g(x)}{z-x} d x
$$

primarily defined on integrable $2 \mathrm{D}$ vector-functions.

Lemma 3.1. Let $J$ be of Szegö class and $\mathcal{F}^{ \pm}$give its representations as the operator multiplication by $z(t)$ in the model spaces $L_{s_{ \pm}}^{2}$. Then

$$
\left[\begin{array}{l}
\mathcal{F}^{-} f^{-} \\
\mathcal{F}^{+} f^{+}
\end{array}\right](\zeta)=p_{0} \Phi(\zeta)\{\mathfrak{H}(\rho \hat{f})\}(z(\zeta))
$$


for any finite vector $f=f^{-} \oplus f^{+} \in l^{2}(\mathbb{Z})=l^{2}\left(\mathbb{Z}_{-}\right) \oplus l^{2}\left(\mathbb{Z}_{+}\right)$.

Proof. Let $\tilde{\mathcal{P}}_{n}(z)$ denote the $n$-th matrix orthonormal polynomial with respect the spectral measure $d \sigma$. Recall, that

$$
\tilde{\mathcal{P}}_{n}(z)=\left[\begin{array}{ll}
\hat{e}_{-n-1}(z) & \hat{e}_{n}(z)
\end{array}\right]=\left[\begin{array}{cc}
P_{n}^{-}(z) & -p_{0} Q_{n}^{+}(z) \\
-p_{0} Q_{n}^{-}(z) & P_{n}^{-}(z)
\end{array}\right]
$$

and, analogically to the scalar case,

$$
\tilde{\mathcal{Q}}_{n}(z):=\int d \sigma(x) \frac{\tilde{\mathcal{P}}_{n}(z)-\tilde{\mathcal{P}}_{n}(x)}{z-x}=\left[\begin{array}{cc}
Q_{n}^{-}(z) & 0 \\
0 & Q_{n}^{+}(z)
\end{array}\right] .
$$

Based on (3.3), we have

$$
\begin{aligned}
p_{0} \Phi(\zeta) \int d \sigma(x) \frac{\tilde{\mathcal{P}}_{n}(x)}{z(\zeta)-x} & =p_{0} \Phi(\zeta)\left\{\int \frac{d \sigma(x)}{z(\zeta)-x} \tilde{\mathcal{P}}_{n}(z(\zeta))-\int d \sigma(x) \frac{\tilde{\mathcal{P}}_{n}(z(\zeta))-\tilde{\mathcal{P}}_{n}(x)}{z(\zeta)-x}\right\} \\
& =-p_{0} \Phi(\zeta) R(z(\zeta)) \tilde{\mathcal{P}}_{n}(z(\zeta))-p_{0} \Phi(\zeta) \tilde{\mathcal{Q}}_{n}(z(\zeta)) .
\end{aligned}
$$

Using (2.8) and definition (2.10), we get

$$
\begin{aligned}
\left.p_{0} \Phi(\zeta) \int \frac{\rho(x)\left[\hat{e}_{-n-1}(x)\right.}{z(\zeta)-x} \hat{e}_{n}(x)\right] & =\Psi(\zeta) \tilde{\mathcal{P}}_{n}(z(\zeta))-p_{0} \Phi(\zeta) \tilde{\mathcal{Q}}_{n}(z(\zeta)) \\
& =\left[\begin{array}{cc}
e^{-}(n, \zeta) & 0 \\
0 & e^{+}(n, \zeta)
\end{array}\right] \\
& =\left[\begin{array}{cc}
\left(\mathcal{F}^{-} e_{-n-1}\right)(\zeta) & 0 \\
0 & \left(\mathcal{F}^{+} e_{n}\right)(\zeta)
\end{array}\right] .
\end{aligned}
$$

In fact, this finishes the proof.

Theorem 3.1. Let $\rho(x)$ be the spectral density of a Jacobi matrix $J$ of Szegö class and $s_{+}(t)$ be the reflection coefficient. Then the following statements are equivalent:

1. There exist $C<\infty$ such that

$$
\begin{aligned}
& \int_{E}(\mathfrak{H} g)^{*}(x-i 0) \rho^{-1}(x)(\mathfrak{H} g)(x-i 0) d x+\int_{E}(\mathfrak{H} g)^{*}(x+i 0) \rho^{-1}(x)(\mathfrak{H} g)(x+i 0) d x \\
& \leq C \int_{E} g^{*}(x) \rho^{-1}(x) g(x) d x .
\end{aligned}
$$

2. $s_{+}$determines $J$ and the operators $\left(I+\mathcal{H}_{s_{ \pm}}\right)$are invertible. 
Proof. $1 \Rightarrow 2$. Since (see (1.30))

$$
\begin{aligned}
\left\|\mathcal{F}^{-} f^{-}\right\|^{2}+\left\|\mathcal{F}^{+} f^{+}\right\|^{2} & =\int_{\mathbb{E}}\{\mathfrak{H}(\rho \hat{f})\}^{*}(z(t))\left(p_{0} \Phi\right)^{*}(t)\left(p_{0} \Phi\right)(t)\{\mathfrak{H}(\rho \hat{f})\}(z(t)) d m \\
& =\int_{\mathbb{E}}\{\mathfrak{H}(\rho \hat{f})\}^{*}(z(t)) \frac{1}{2 \pi} \rho^{-1}(z(t))\{\mathfrak{H}(\rho \hat{f})\}(z(t)) \frac{\left|z^{\prime}(t) \| d t\right|}{2 \pi} \\
& =\left(\frac{1}{2 \pi}\right)^{2} \int_{E}\{\mathfrak{H}(\rho \hat{f})\}^{*}(x-i 0) \rho^{-1}(x)\{\mathfrak{H}(\rho \hat{f})\}(x-i 0) d x \\
& +\left(\frac{1}{2 \pi}\right)^{2} \int_{E}\{\mathfrak{H}(\rho \hat{f})\}^{*}(x+i 0) \rho^{-1}(x)\{\mathfrak{H}(\rho \hat{f})\}(x+i 0) d x \\
& \leq \frac{C}{(2 \pi)^{2}} \int_{E} \hat{f}^{*}(x) \rho(x) \hat{f}(x) d x=\frac{C}{(2 \pi)^{2}}\|f\|^{2},
\end{aligned}
$$

we get $\mathcal{F}^{ \pm} f^{ \pm} \in A_{1}^{2}\left(\Gamma, \alpha_{ \pm}\right)$. Thus, $\mathcal{F}^{ \pm}\left\{l^{2}\left(Z_{ \pm}\right)\right\}=H_{s_{ \pm}}^{2}\left(\alpha_{ \pm}\right)$. By Lemma 1.7 and Theorem 2.1 we come to the conclusion that $s_{+}$determines $J$. Further, by (3.5)

$$
\begin{aligned}
\left\|\mathcal{F}^{-} f^{-}\right\|^{2}+\left\|\mathcal{F}^{+} f^{+}\right\|^{2} & \leq \frac{C}{(2 \pi)^{2}}\left\{\left\|f^{-}\right\|^{2}+\left\|f^{+}\right\|^{2}\right\} \\
& =\frac{C}{(2 \pi)^{2}}\left\{\left\|\mathcal{F}^{-} f^{-}\right\|_{s_{-}}^{2}+\left\|\mathcal{F}^{+} f^{+}\right\|_{s_{+}}^{2}\right\} .
\end{aligned}
$$

Using again $\mathcal{F}^{ \pm} f^{ \pm} \in A_{1}^{2}\left(\Gamma, \alpha_{ \pm}\right)$, we can represent the last norms in the form

$$
\left\|\mathcal{F}^{-} f^{-}\right\|^{2}+\left\|\mathcal{F}^{+} f^{+}\right\|^{2} \leq \frac{C}{(2 \pi)^{2}}\left\{\left\langle\left(I+\mathcal{H}_{s_{-}}\right) \mathcal{F}^{-} f^{-}, \mathcal{F}^{-} f^{-}\right\rangle+\left\langle\left(I+\mathcal{H}_{s_{+}}\right) \mathcal{F}^{+} f^{+}, \mathcal{F}^{+} f^{+}\right\rangle\right\} .
$$

This proves the second statement in 2 .

$2 \Rightarrow 1$. Recall that $H_{s_{ \pm}}^{2}\left(\alpha_{ \pm}\right)=\cos _{L_{s_{ \pm}}^{2}} A_{1}^{2}\left(\Gamma, \alpha_{ \pm}\right)$, but in the case under consideration, the norm in $H_{s_{ \pm}}^{2}\left(\alpha_{ \pm}\right)$is equivalent to the norm in $A_{1}^{2}\left(\Gamma, \alpha_{ \pm}\right)$, i.e.:

$$
h \in H_{s_{ \pm}}^{2}\left(\alpha_{ \pm}\right) \Rightarrow h \in A_{1}^{2}\left(\Gamma, \alpha_{ \pm}\right) .
$$

Further, since $s_{+}$determines $J$, by Lemma 1.7 , we have $\mathcal{F}^{ \pm}\left\{l^{2}\left(Z_{ \pm}\right)\right\}=H_{s_{ \pm}}^{2}\left(\alpha_{ \pm}\right)$. So, starting with (3.6) we obtain (3.4).

Remark on ISP and RHP. Let us define $\sqrt{-b^{2} z^{\prime}}(\zeta)$ as the square root of an outer function such that $\sqrt{-b^{2} z^{\prime}}(0)>0$. Put

$$
\sqrt{-z^{\prime}}(\zeta)=\frac{\sqrt{-b^{2} z^{\prime}}(\zeta)}{b(\zeta)}
$$

In this case, $\overline{\sqrt{-z^{\prime}}(\bar{\zeta})}=\sqrt{-z^{\prime}}(\zeta)$. Let $\mathbb{E}_{-}=\{t \in \mathbb{E}: \operatorname{Im} t<0\}$. Then $-t z^{\prime}(t)=i\left|z^{\prime}(t)\right|$, $t \in \mathbb{E}_{-}$. Thus,

$$
t\left\{\sqrt{-z^{\prime}}(t)\right\}^{2}=i \sqrt{-z^{\prime}}(t) \overline{\sqrt{-z^{\prime}}(t)}=i \sqrt{-z^{\prime}}(t) \sqrt{-z^{\prime}}(\bar{t}), \quad t \in \mathbb{E}_{-},
$$


or

$$
\bar{t} \sqrt{-z^{\prime}}(\bar{t})=-i \sqrt{-z^{\prime}}(t), \quad t \in \mathbb{E}_{-} .
$$

Besides,

$$
\sqrt{-z^{\prime}} \mid[\gamma]=\epsilon(\gamma) \sqrt{-z^{\prime}}(\zeta)
$$

where $\epsilon \in \Gamma^{*}, \epsilon^{2}=1_{\Gamma^{*}}$. But, in fact, the group $\Gamma$ is defined up to a choice of a half-period $\tilde{\epsilon} \in \Gamma^{*}$. So, we may assume that

$$
\sqrt{-z^{\prime}} \mid[\gamma]=\sqrt{-z^{\prime}}(\zeta)
$$

Lemma 4.1. Let $E=\left[b_{0}, a_{0}\right] \backslash \cup_{j \geq 1}\left(a_{j}, b_{j}\right)$ be a homogeneous set. Then

$$
\Pi(z(\zeta)):=\frac{\Phi(\zeta)}{\sqrt{-z^{\prime}}(\zeta)}
$$

is a holomorphic matrix function in $\overline{\mathbb{C}} \backslash\left[b_{0}, a_{0}\right]$ satisfying the following $R H P$

$$
\begin{aligned}
& \Pi(x-i 0)=\left[\begin{array}{cc}
\alpha_{-, j} & 0 \\
0 & \alpha_{+, j}
\end{array}\right] \Pi(x+i 0), \quad x \in\left(a_{j}, b_{j}\right), \\
& \Pi(x-i 0)=-i \Sigma(x) \Pi(x+i 0), \quad x \in E,
\end{aligned}
$$

where $\Sigma(z(t)):=S(t), t \in \mathbb{E}_{-}$, with the normalization at infinity:

$$
\Pi(z)=\left[\begin{array}{cc}
1+\ldots & -\frac{a}{z}+\ldots \\
-\frac{b}{z}+\ldots & 1+\ldots
\end{array}\right]\left[\begin{array}{cc}
\frac{1}{\sqrt{b s(0)}} & 0 \\
0 & \frac{1}{\sqrt{a s(0)}}
\end{array}\right] .
$$

Proof. (4.4) follows from (4.3) and (4.5) follows from (4.2) and (2.6). To prove (4.6), we represent $\Pi(z)$ in the form

$$
\Pi(z)=\left[\begin{array}{cc}
1+\ldots & -\frac{a}{z}+\ldots \\
-\frac{b}{z}+\ldots & 1+\ldots
\end{array}\right]\left[\begin{array}{cc}
c_{1} & 0 \\
0 & c_{2}
\end{array}\right]
$$

Then, we note that

$$
c_{1} c_{2}=\operatorname{det} \Pi(\infty)=\left.\frac{e^{-}(-1, \zeta) e^{+}(-1, \zeta)-e^{-}(0, \zeta) e^{+}(0, \zeta)}{-z^{\prime}(\zeta)}\right|_{\zeta=0}=\frac{1}{p_{0} s(0)},
$$

and

$$
\frac{a c_{2}}{c_{1}}=\frac{b c_{1}}{c_{2}}=\left.\frac{z(\zeta) e^{ \pm}(0, \zeta)}{e^{ \pm}(-1, \zeta)}\right|_{\zeta=0}=p_{0} .
$$

Solving together (4.7), (4.8), we get (4.6). 


\section{Matrix $A_{2}$ ON homogeneOus Sets}

Let $E$ be a homogeneous set. Throughout this section $P_{+}$denotes orthoprojector from the vector-valued $L^{2}\left(\mathbb{C}^{n}\right)$ onto $H^{2}\left(\mathbb{C}^{n}\right)$ in the upper halfplane. We are interested in the boundedness of the weighted transform

$$
W^{1 / 2} P_{+} W^{-1 / 2}: \chi_{E} L^{2}\left(\mathbb{C}^{n}\right) \rightarrow \chi_{E} L^{2}\left(\mathbb{C}^{n}\right),
$$

where $W$ is a weight on $E$ and $\chi_{E}$ is the characteristic function of the set $E$.

Here is an analog of the matrix $A_{2}$ condition

$$
\sup _{x \in E, 0<\delta<1} \|\langle W\rangle_{I_{(x, \delta)}^{1 / 2}}^{I^{-1}}\left\langle W^{-1 / 2} \|<\infty\right.
$$

where $I_{(x, \delta)}:=(x-\delta, x+\delta)$ and

$$
\langle W\rangle_{I_{(x, \delta)}}:=\frac{1}{\left|I_{(x, \delta)}\right|} \int_{I_{(x, \delta)} \cap E} W(t) d t .
$$

This supremum will be called $Q_{2, E}(W)$.

Theorem 5.1. The operator (5.1) is bounded if and only if $Q_{2, E}(W)<\infty$.

Proof of necessity. With an arbitrary $z_{0} \in \mathbb{C}_{+}$we associate a subspace $K_{b_{z_{0}}}=H^{2}\left(\mathbb{C}^{n}\right) \ominus$ $b_{z_{0}} H^{2}\left(\mathbb{C}^{n}\right)$ of the Hardy space, $b_{z_{0}}(z)=\frac{z-z_{0}}{z-z_{0}}$. It is well known, that

$$
P_{K_{b_{0}}}=P_{+}-b_{z_{0}} P_{+} \overline{b_{z_{0}}}
$$

and

$$
\left\langle P_{K_{b_{0}}} f, g\right\rangle_{L^{2}\left(\mathbb{C}^{n}\right)}=\left\langle\left(P_{+} f\right)\left(z_{0}\right),\left(P_{+} g\right)\left(z_{0}\right)\right\rangle_{C^{n}}
$$

Because of the first of these relations we have

$$
\left|\left\langle W^{1 / 2} P_{K_{b_{0}}} W^{-1 / 2} f, g\right\rangle\right| \leq 2 Q\left\|\chi_{E} f\right\|\left\|\chi_{E} g\right\| .
$$

Now, using the second one we get

$$
\left|\left\langle\left(P_{+} W^{-1 / 2} f\right)\left(z_{0}\right),\left(P_{+} W^{1 / 2} g\right)\left(z_{0}\right)\right\rangle\right| \leq 2 Q\left\|\chi_{E} f\right\|\left\|\chi_{E} g\right\| .
$$

Let us substitute

$$
f=W^{-1 / 2} \frac{\xi}{x-\overline{z_{0}}}, \quad g=W^{1 / 2} \frac{\eta}{x-\overline{z_{0}}}, \quad \xi, \eta \in \mathbb{C}^{n}
$$

in (5.3). This give us

$$
\left|\left\langle\left\langle W^{-1}\right\rangle_{z_{0}} \xi,\langle W\rangle_{z_{0}} \eta\right\rangle\right| \leq 2 Q\left\|\left\langle W^{-1}\right\rangle_{z_{0}}^{1 / 2} \xi\right\|\left\|\langle W\rangle_{z_{0}}^{1 / 2} \eta\right\|,
$$

where $\langle W\rangle_{z_{0}}$ denotes an average with the Poisson kernel,

$$
\langle W\rangle_{z_{0}}:=\frac{1}{\pi} \int W \frac{\operatorname{Im} z_{0}}{\left|x-z_{0}\right|^{2}} d x .
$$

Thus we proved an inequality with the Poisson's averages

$$
\langle W\rangle_{z_{0}} \leq 2 Q\left\langle W^{-1}\right\rangle_{z_{0}}^{-1}
$$

At last let us note that

$$
\frac{\operatorname{Im} z_{0}}{\left|x-z_{0}\right|^{2}} \geq \frac{c}{|I|} \chi_{I}, \quad I=I_{\left(\operatorname{Re} z_{0}, \operatorname{Im} z_{0}\right)},
$$

with an absolute and positive constant $c$. Therefore (5.4) implies (5.2). 
Lemma 5.1. If $I$ is a centered at $E$ interval and $z_{0}$ is the center of the square built on $I$, then

$$
W \in A_{2}(E) \Rightarrow\langle W\rangle_{z_{0}} \leq C\left(E, Q_{2, E}(W)\right)\langle W\rangle_{I}
$$

Proof. First we note, that for $\lambda=2 / \eta$

$$
|\lambda I \cap E| \geq \eta|\lambda I| \geq 2|I| \geq 2|I \cap E|
$$

and therefore $|(\lambda I \backslash I) \cap E| \geq|I \cap E|$. Let us show that

$$
W(\lambda I) \geq\left(1+\frac{\eta^{2}}{\lambda^{2} Q^{2}}\right) W(I) \quad \text { for } W \in A_{2}(E) .
$$

Integrating the inequality

$$
\left[\begin{array}{cc}
W^{-1} & 1 \\
1 & W
\end{array}\right] \geq 0
$$

over $(\lambda I \backslash I) \cap E$ we get

$$
\left[\begin{array}{cc}
W^{-1}(\lambda I \backslash I) & |(\lambda I \backslash I) \cap E| \\
|(\lambda I \backslash I) \cap E| & W(\lambda I \backslash I)
\end{array}\right] \geq 0
$$

Therefore

$$
\left[\begin{array}{cc}
W^{-1}(\lambda I) & |(\lambda I \backslash I) \cap E| \\
|(\lambda I \backslash I) \cap E| & W(\lambda I)-W(I)
\end{array}\right] \geq 0,
$$

or

$$
W(\lambda I)-W(I) \geq|(\lambda I \backslash I) \cap E|^{2}\left\{W^{-1}(\lambda I)\right\}^{-1} \geq|I \cap E|^{2}\left\{W^{-1}(\lambda I)\right\}^{-1} .
$$

Using (5.2) we obtain

$$
W(\lambda I)-W(I) \geq \frac{|I \cap E|^{2}}{Q^{2}|\lambda I|^{2}} W(\lambda I) \geq \frac{\eta^{2}}{Q^{2} \lambda^{2}} W(I) .
$$

To prove (5.5), using

$$
\frac{\operatorname{Im} z_{0}}{\left|x-z_{0}\right|^{2}} \leq \frac{c}{|I|} \sum \frac{1}{\lambda^{2 k}} \chi_{\lambda^{k} I}, \quad \lambda^{k} I=I_{\left(\operatorname{Re} z_{0}, \lambda^{k} \operatorname{Im} z_{0}\right)}
$$

we write the following chain of inequalities:

$$
\begin{aligned}
\langle W\rangle_{z_{0}} & \leq \frac{c}{|I|} \sum \frac{1}{\lambda^{2 k}} W\left(\lambda^{k} I\right) \\
& \leq \frac{c}{|I|} \sum \frac{Q^{2}\left|\lambda^{k} I\right|^{2}}{\lambda^{2 k}}\left\{W^{-1}\left(\lambda^{k} I\right)\right\}^{-1} \\
& \leq \frac{c Q^{2}}{|I|} \sum\left(1+\frac{\eta^{2}}{\lambda^{2} Q^{2}}\right)^{-k}|I|^{2}\left\{W^{-1}(I)\right\}^{-1} \\
& \leq \frac{c Q^{2}}{\eta^{2}} \sum\left(1+\frac{\eta^{2}}{\lambda^{2} Q^{2}}\right)^{-k}\langle W\rangle_{I} .
\end{aligned}
$$


Proof of sufficiency. We want to prove that (5.2) suffices for $W^{1 / 2} P_{+} W^{-1 / 2}$ in (5.1) to be bounded. Fix $f, g \in \chi_{E} L^{2}\left(\mathbb{C}^{n}\right)$. We need to show

$$
\int_{\mathbb{C}_{+}}\left|\left\langle\left(P_{+} W^{-1 / 2} f\right)^{\prime}(z),\left(P_{+} W^{1 / 2} g\right)^{\prime}(z)\right\rangle_{\mathbb{C}^{n}}\right| \operatorname{Im} z d A(z) \leq C\|f\|\|g\| .
$$

In other words, introducing a Stolz cone $\Gamma_{t}$ and

$$
S(t)=\int_{\Gamma_{t}}\left|\left\langle\left(P_{+} W^{-1 / 2} f\right)^{\prime}(z),\left(P_{+} W^{1 / 2} g\right)^{\prime}(z)\right\rangle_{\mathbb{C}^{n}}\right| d A(z)
$$

one needs to prove that

$$
\int S(t) d t \leq C\|f\|\|g\|
$$

We follow closely the lines of the proof in [10]. Let us consider a nonnegative function $h(t)$ and

$$
S_{h(t)}(t)=\int_{\Gamma_{t, h(t)}}\left|\left\langle\left(P_{+} W^{-1 / 2} f\right)^{\prime}(z),\left(P_{+} W^{1 / 2} g\right)^{\prime}(z)\right\rangle_{\mathbb{C}^{n}}\right| d A(z),
$$

where

$$
\Gamma_{t, h(t)}=\Gamma_{t} \cap\{z: \operatorname{Im} z \leq h(t)\}
$$

Let us note that

$$
\int S(t) d t \leq c \int S_{h(t)} d t
$$

if the function $h(t)$ has the following property:

$$
\forall I \subset \mathbb{R} \quad|\{t \in I: h(t) \geq|I|\}| \geq a|I| .
$$

Let us choose $h$ to be maximal such that

$$
S_{h(t)}(t) \leq B\left(M\|f\|^{p_{*}}\right)^{1 / p_{*}}(t)\left(M\|g\|^{p_{*}}\right)^{1 / p_{*}}(t),
$$

where $B, p_{*} \in(1,2)$ will be chosen a bit later and $M$ denotes the maximal function

$$
(M f)(x)=\sup _{\delta>0} \frac{1}{|I(x, \delta)|} \int_{I(x, \delta)}|f(t)| d t .
$$

If this $h$ satisfies (5.10), then (5.9) and (5.8) imply what we need.

To choose $B, p_{*}$ and to prove that $h$ satisfies (5.9) we follow the algorithm below. Let $I_{0}$ be an arbitrary interval on the real axis. We will consider two cases: $2 I_{0} \cap E \neq \emptyset$ and $2 I_{0} \cap E=\emptyset$.

In the first case we fix an interval $I$ centered at $E$ such that $I_{0} \subset I$ and $|I| \leq 3\left|I_{0}\right|$. Let $f_{1}=f \cdot \chi_{2 I}, g_{1}=g \cdot \chi_{2 I}$ and $f_{2}=f-f_{1}, g_{2}=g-g_{1}$. Denote $A_{I}=\langle W\rangle_{I}^{1 / 2}$. Consider

$$
\begin{aligned}
& t \in I, \quad S^{A_{I}}\left(f_{i}\right)(t)=\left(\int_{\Gamma_{t,|I|}}\left\|\left(P_{+} A_{I} W^{-1 / 2} f_{i}\right)^{\prime}\right\|^{2} d A(z)\right)^{1 / 2}, \quad i=1,2 \\
& t \in I, \quad S^{A_{I}^{-1}}\left(g_{i}\right)(t)=\left(\int_{\Gamma_{t,|I|}}\left\|\left(P_{+} A_{I}^{-1} W^{1 / 2} g_{i}\right)^{\prime}\right\|^{2} d A(z)\right)^{1 / 2}, \quad i=1,2 .
\end{aligned}
$$


We will fix later $\alpha=\alpha\left(Q_{2, E}(W), n\right)>1$. Now,

$$
\begin{aligned}
& \frac{1}{|I|} \int_{I}\left(S^{A_{I}^{-1}}\left(g_{1}\right)(t)\right)^{\alpha} d t \leq \frac{C(\alpha)}{|I|} \int_{2 I}\left\|A_{I}^{-1} W^{1 / 2} g(t)\right\|^{\alpha} d t \\
& \leq \frac{C_{1}(\alpha, n)}{|I|} \int_{2 I}\left(\|g(t)\| \sum_{i=1}^{n}\left\|W^{1 / 2}(t) A_{I}^{-1} e_{i}\right\|\right)^{\alpha} d t \\
& \leq \frac{C_{2}(\alpha, n)}{|I|}\left(\int_{2 I}\|g(t)\|^{(2-\tilde{\epsilon}) \alpha} d t\right)^{\frac{1}{2-\tilde{\epsilon}}} \sum_{i=1}^{n}\left(\int_{2 I}\left\|W^{1 / 2}(t) A_{I}^{-1} e_{i}\right\|^{(2+\epsilon) \alpha} d t\right)^{\frac{1}{2+\epsilon}} \text {. }
\end{aligned}
$$

Here $(2+\epsilon)^{-1}+(2-\tilde{\epsilon})^{-1}=1$. Notice that for every vector $x \in \mathbb{C}^{n}$ the scalar function $t \rightarrow\left\|W(t)^{1 / 2} x\right\|$ is uniformly in scalar $A_{2}(E)$. In particular, there exists such an $\epsilon_{0}>0$ that we have the inverse Hölder inequality for all such functions uniformly:

$\forall I$ centered at $x \in E \quad\left(\frac{1}{|I|} \int_{I}\left\|W(t)^{1 / 2} x\right\|^{2+\epsilon_{0}} d t\right)^{\frac{1}{2+\epsilon_{0}}} \leq C\left(\frac{1}{|I|} \int_{I}\left\|W(t)^{1 / 2} x\right\|^{2} d t\right)^{\frac{1}{2}}$.

Let us choose $\epsilon=\frac{\epsilon_{0}}{2}\left(\tilde{\epsilon}=\frac{\epsilon_{0}}{2+\epsilon_{0}}\right), \alpha=1+\frac{\epsilon_{0}}{2\left(2+\epsilon_{0}\right)}$, then we have

$$
\begin{gathered}
(2+\epsilon) \alpha<2+\epsilon_{0}, \\
(2-\tilde{\epsilon}) \alpha<2 .
\end{gathered}
$$

We use (5.13) and inverse Hölder inequality (5.12) in (5.11) to rewrite it as

$$
\begin{aligned}
& \left(\frac{1}{|I|} \int_{I}\left(S^{A_{I}^{-1}}\left(g_{1}\right)(t)\right)^{\alpha} d t\right)^{1 / \alpha} \\
& \leq C(\alpha, n)\left(\frac{1}{|I|} \int_{2 I}\|g(t)\|^{(2-\tilde{\epsilon}) \alpha} d t\right)^{\frac{1}{(2-\tilde{\epsilon}) \alpha}} \sum_{i=1}^{n}\left(\frac{1}{|I|} \int_{2 I}\left\|W^{1 / 2}(t) A_{I}^{-1} e_{i}\right\|^{2} d t\right)^{\frac{1}{2}} \\
& \leq C_{1}(\alpha, n)\left(\frac{1}{|I|} \int_{2 I}\|g(t)\|^{(2-\tilde{\epsilon}) \alpha} d t\right)^{\frac{1}{(2-\tilde{\epsilon}) \alpha}} \sum_{i=1}^{n}\left\langle\langle W\rangle_{I}^{-1 / 2}\langle W\rangle_{2 I}\langle W\rangle_{I}^{-1 / 2} e_{i}, e_{i}\right\rangle^{\frac{1}{2}} \\
& \leq C_{3}\left(\alpha, n, Q_{2, E}(W)\right) \inf _{x \in I}\left(M\|g\|^{p_{*}}\right)^{\frac{1}{p_{*}}}(x),
\end{aligned}
$$

where $p_{*}=(2-\tilde{\epsilon}) \alpha<2$. We used the doubling property of $W:\langle W\rangle_{I}^{-1 / 2}\langle W\rangle_{2 I}\langle W\rangle_{I}^{-1 / 2} \leq$ $2 \frac{Q^{2}}{\eta^{2}}$, the inequality which can be proved in the same way as (5.6).

The last inequality ensures that for any $\tau, \tau \in(0,1)$, using Kolmogorov-type inequalities we can find a subset $E\left(\tau, I_{0}\right) \subset I_{0},\left|E\left(\tau, I_{0}\right)\right| \geq\left|I_{0}\right|-\tau^{\alpha}|I| \geq\left(1-3 \tau^{\alpha}\right)\left|I_{0}\right|$ such that

$$
t \in E\left(\tau, I_{0}\right) \Rightarrow \quad S^{A_{I}^{-1}}\left(g_{1}\right)(t) \leq \frac{C_{3}\left(\alpha, n, Q_{2, E}(W)\right)}{\tau} \inf _{x \in I}\left(M\|g\|^{p_{*}}\right)^{\frac{1}{p_{*}}}(x) .
$$

Similarly, for every $\tau$ there exists a set $E\left(\tau, I_{0}\right),\left|E\left(\tau, I_{0}\right)\right| \geq\left(1-3 \tau^{\alpha}\right)\left|I_{0}\right|$ such that

$$
t \in E\left(\tau, I_{0}\right) \Rightarrow \quad S^{A_{I}}\left(f_{1}\right)(t) \leq \frac{C\left(\alpha, n, Q_{2, E}(W)\right)}{\tau} \inf _{x \in I}\left(M\|f\|^{p_{*}}\right)^{\frac{1}{p_{*}}}(x) .
$$


Here we use the same calculations and the fact that for any $I$ centered at $E$

$$
\langle W\rangle_{I}^{1 / 2}\left\langle W^{-1}\right\rangle_{2 I}\langle W\rangle_{I}^{1 / 2} \leq 2 Q^{2} .
$$

Now let us work with $f_{2}, g_{2}$. Let $c_{I}$ be the center of the square built on $2 I$. Using the representation

$$
\left(P_{+} A_{I} W^{-1 / 2} f_{2}\right)^{\prime}(z)=\frac{1}{2 \pi i} \int \frac{\left(A_{I} W^{-1 / 2} f_{2}\right)(x)}{(x-z)^{2}} d x, \quad \operatorname{Im} z>0,
$$

clearly, we obtain for every $t \in I$

$$
\left(\int_{\Gamma_{t,|I|}}\left\|\left(P_{+} A_{I} W^{-1 / 2} f_{2}\right)^{\prime}(z)\right\|^{2} d A(z)\right)^{1 / 2} \leq C \int \frac{\operatorname{Im} c_{I}}{\left|x-c_{I}\right|^{2}}\left\|\left(A_{I} W^{-1 / 2} f_{2}\right)(x)\right\| d x .
$$

Therefore, using the inverse Hölder inequality (5.12), we have again

$$
\begin{aligned}
& \left(\int_{\Gamma_{t,|I|}}\left\|\left(P_{+} A_{I} W^{-1 / 2} f_{2}\right)^{\prime}(z)\right\|^{2} d A(z)\right)^{1 / 2} \leq C \sum_{i=1}^{n} \int \frac{\operatorname{Im} c_{I}}{\left|x-c_{I}\right|^{2}}\left\|W^{-1 / 2} A_{I} e_{i}\right\|\left\|f_{2}\right\| d x \\
& \leq C_{1} \sum_{i=1}^{n}\left(\int \frac{\operatorname{Im} c_{I}}{\left|x-c_{I}\right|^{2}}\left\|W^{-1 / 2} A_{I} e_{i}\right\|^{2+\epsilon} d x\right)^{\frac{1}{2+\epsilon}}\left(\int \frac{\operatorname{Im} c_{I}}{\left|x-c_{I}\right|^{2}}\left\|f_{2}\right\|^{2-\tilde{\epsilon}} d x\right)^{\frac{1}{2-\tilde{\epsilon}}} \\
& \leq C_{2} \sum_{i=1}^{n}\left\langle\langle W\rangle_{I}^{1 / 2}\left\langle W^{-1}\right\rangle_{c_{I}}\langle W\rangle_{I}^{1 / 2} e_{i}, e_{i}\right\rangle^{\frac{1}{2}} \inf _{x \in I}\left(M\|f\|^{2-\tilde{\epsilon}}(x)\right)^{\frac{1}{2-\epsilon}}
\end{aligned}
$$

Here $2+\epsilon$ is close to $2\left(\epsilon \leq \epsilon_{0}\right)$. Finally, using Lemma 5.1 we estimate the last sum by a constant:

$$
\left(\int_{\Gamma_{t,|I|}}\left\|\left(P_{+} A_{I} W^{-1 / 2} f_{2}\right)^{\prime}(z)\right\|^{2} d A(z)\right)^{1 / 2} \leq C(n, E, Q) \inf _{x \in I}\left(M\|f\|^{2-\tilde{\epsilon}}(x)\right)^{\frac{1}{2-\epsilon}} .
$$

That is

$$
S^{A_{I}}\left(f_{2}\right)(t) \leq C(n, E, Q) \inf _{x \in I}\left(M\|f\|^{2-\tilde{\epsilon}}(x)\right)^{\frac{1}{2-\tilde{\epsilon}}}, \quad \forall t \in I .
$$

The same for $S^{A_{I}^{-1}}\left(g_{2}\right)(t)$.

Combining all $(*)$ inequalities we obtain that with a suitable $C=C(n, E, W)$

$$
\begin{aligned}
S_{I}(t) & :=\int_{\Gamma_{t,|I|}}\left|\left\langle\left(P_{+} W^{-1 / 2} f\right)^{\prime}(z),\left(P_{+} W^{1 / 2} g\right)^{\prime}(z)\right\rangle_{\mathbb{C}^{n}}\right| d A(z) \\
& \leq S^{A_{I}}(f)(t) S^{A_{I}^{-1}}(g)(t) \leq C^{2}\left(M \| f||^{p_{*}}(t)\right)^{\frac{1}{p_{*}}}\left(M\|g\|^{p_{*}}(t)\right)^{\frac{1}{p_{*}}}
\end{aligned}
$$

at least on a quarter of $I_{0}$. Of course, $S_{I_{0}}(t) \leq S_{I}(t)$. 
In the case $2 I_{0} \cap E=\emptyset$ we fix an interval $I$ centered at $E$ such that $I_{0} \subset I$ and $\operatorname{dist}\left(I_{0}, E\right) \geq|I| / 6$. Let $c_{I}$ be the center of the square built on $I$. We can use again a representation of the form (5.15):

$$
\left(P_{+} A_{I} W^{-1 / 2} f\right)^{\prime}(z)=\frac{1}{2 \pi i} \int \frac{\left(A_{I} W^{-1 / 2} f\right)(x)}{(x-z)^{2}} d x, \quad \operatorname{Im} z>0,
$$

to obtain an analog of $(5.16)$

$$
\left(\int_{\Gamma_{t,|I|}}\left\|\left(P_{+} A_{I} W^{-1 / 2} f\right)^{\prime}(z)\right\|^{2} d A(z)\right)^{1 / 2} \leq C \int \frac{\operatorname{Im} c_{I}}{\left|x-c_{I}\right|^{2}}\left\|\left(A_{I} W^{-1 / 2} f\right)(x)\right\| d x
$$

for all $t \in I_{0}$. Continue in this way we get

$$
S^{A_{I}}(f)(t) \leq C(n, E, Q) \inf _{x \in I}\left(M\|f\|^{p_{*}}(x)\right)^{\frac{1}{p_{*}}}, \quad \forall t \in I_{0} .
$$

The same for $S^{A_{I}^{-1}}(g)(t)$. Thus

$$
S_{I}(t) \leq C(n, E, Q)^{2}\left(M\|f\|^{p_{*}}(t)\right)^{\frac{1}{p_{*}}}\left(M\|g\|^{p_{*}}(t)\right)^{\frac{1}{p_{*}}}
$$

everywhere on $I_{0}$.

Let $B$ be the largest constant in (5.18), (5.19). We have already chosen $p_{*}<2$. Now we introduce the following function $h(t)$ :

$$
h(t)=\sup \left\{h: S_{h}(t) \leq B\left(M\|f\|^{p_{*}}(t)\right)^{\frac{1}{p_{*}}}\left(M\|g\|^{p_{*}}(t)\right)^{\frac{1}{p_{*}}}\right\} .
$$

What we proved can be summarized in:

$$
\begin{aligned}
& \text { if } I_{0}: 2 I_{0} \cap E \neq \emptyset \text { then } h(t) \geq\left|I_{0}\right| \quad \text { on a quarter of measure of } I_{0} \text {, } \\
& \text { if } I_{0}: 2 I_{0} \cap E=\emptyset \text { then } h(t) \geq\left|I_{0}\right| \quad \forall t \in I_{0} \text {. }
\end{aligned}
$$

In any case,

$$
\begin{aligned}
& \frac{1}{4} \int_{\mathbb{C}_{+}}\left|\left\langle\left(P_{+} W^{-1 / 2} f\right)^{\prime}(z),\left(P_{+} W^{1 / 2} g\right)^{\prime}(z)\right\rangle_{\mathbb{C}^{n}}\right| \operatorname{Im} z d A(z) \\
& \leq \int_{\mathbb{R}} \int_{\Gamma_{t, h(t)}}\left|\left\langle\left(P_{+} W^{-1 / 2} f\right)^{\prime}(z),\left(P_{+} W^{1 / 2} g\right)^{\prime}(z)\right\rangle_{\mathbb{C}^{n}}\right| d A(z) d t \\
& \leq B \int_{\mathbb{R}}\left(M\|f\|^{p_{*}}(t)\right)^{\frac{1}{p_{*}}}\left(M \|\left. g\right|^{p_{*}}(t)\right)^{\frac{1}{p_{*}}} d t \\
& \leq B\left(\int_{\mathbb{R}}\left(M\|f\|^{p_{*}}(t)\right)^{\frac{2}{p_{*}}} d t\right)^{\frac{1}{2}}\left(\int_{\mathbb{R}}\left(M\|g\|^{p_{*}}(t)\right)^{\frac{2}{p_{*}}} d t\right)^{\frac{1}{2}} \\
& \leq B C\left(p_{*}\right)\left(\int_{\mathbb{R}}\|f\|^{2}(t) d t\right)^{\frac{1}{2}}\left(\int_{\mathbb{R}}\|g\|^{2}(t) d t\right)^{\frac{1}{2}}
\end{aligned}
$$

because $\frac{2}{p_{*}}>1$, and we can use the Hardy-Littlewood maximal theorem. The theorem is proved. 


\section{REFERENCES}

1. D. Arov and H. Dym, On matricial Nehari problems, J-inner matrix functions and the Muckenhoupt condition, J. Funct. Anal. (to appear).

2. L. Carleson, On $H^{\infty}$ in multiply connected domains, Conference on harmonic analysis in honor Antoni Zygmund. (eds. W Beckner, et al.). vol. II., Wadsworth, 1983, pp. 349-372.

3. F. Gesztesy, R. Nowell and W. Pötz, One-dimensional scattering for quantum systems with nontrivial spatial asymptotics, Diff. Integral Eqs. 10 (1997), 521-546.

4. G.S. Guseinov, The determination of an infinite Jacobi matrix from the scattering data, Soviet Math. Dokl. 17 (1976), 596-600.

5. M. Hasumi, Hardy Classes on Infinitely Connected Riemann Surfaces, Lecture Notes in Math. 1027, Spinger Verlag, Berlin and New York, 1983.

6. V. Marchenko, Sturm-Liouville Operators and Applications, Birkhäuser, Basel, 1986.

7. V. Marchenko, Nonlinear equations and operator algebras, Translated from the Russian by V. I. Rublinetskiu. Mathematics and its Applications (Soviet Series), 17. D. Reidel Publishing Co., DordrechtBoston, MA, 1988.

8. F. Peherstorfer and P Yuditskii, Asymptotic behavior of polynomials orthonormal on a homogeneous set, submitted.

9. M. Sodin and P. Yuditskii, Almost periodic Jacobi matrices with homogeneous spectrum, infinite dimensional Jacobi inversion, and Hardy spaces of character-automorphic functions, Journ. of Geom. Analysis 7 (1997), 387-435.

10. A. Volberg, Matrix $A_{p}$ weights via S-functions, Journ. Amer. Math. Soc. 10 (1997), 445-466. 